\title{
Flexible Coordinator and Switcher Hubs for Adaptive Task Control
}

\author{
${ }^{\circledR}$ Carrisa V. Cocuzza, ${ }^{1,2}$ Takuya Ito, ${ }^{1,2}{ }^{\circledR}$ Douglas Schultz, ${ }^{1,3}{ }^{\circledR}$ Danielle S. Bassett, ${ }^{4}$ and ${ }^{\circledR}$ Michael W. Cole ${ }^{1}$ \\ ${ }^{1}$ Center for Molecular and Behavioral Neuroscience, Rutgers University, Newark, New Jersey 07102, ${ }^{2}$ Behavioral and Neural Sciences PhD Program, \\ Rutgers University, Newark, New Jersey 07102, ${ }^{3}$ Center for Brain Biology and Behavior, University of Nebraska-Lincoln, Lincoln, Nebraska 68588, \\ and ${ }^{4}$ Departments of Bioengineering, Physics and Astronomy, Electrical and Systems Engineering, and Neurology, University of Pennsylvania, \\ Philadelphia, Pennsylvania 19104
}

Functional connectivity (FC) studies have identified at least two large-scale neural systems that constitute cognitive control networks, the frontoparietal network (FPN) and cingulo-opercular network (CON). Control networks are thought to support goal-directed cognition and behavior. It was previously shown that the FPN flexibly shifts its global connectivity pattern according to task goal, consistent with a "flexible hub" mechanism for cognitive control. Our aim was to build on this finding to develop a functional cartography (a multimetric profile) of control networks in terms of dynamic network properties. We quantified network properties in (male and female) humans using a high-control-demand cognitive paradigm involving switching among 64 task sets. We hypothesized that cognitive control is enacted by the FPN and CON via distinct but complementary roles reflected in network dynamics. Consistent with a flexible "coordinator" mechanism, FPN connections were varied across tasks, while maintaining within-network connectivity to aid cross-region coordination. Consistent with a flexible "switcher" mechanism, CON regions switched to other networks in a task-dependent manner, driven primarily by reduced within-network connections to other CON regions. This pattern of results suggests FPN acts as a dynamic, global coordinator of goal-relevant information, while CON transiently disbands to lend processing resources to other goal-relevant networks. This cartography of network dynamics reveals a dissociation between two prominent cognitive control networks, suggesting complementary mechanisms underlying goal-directed cognition.

Key words: cognitive control; cognitive flexibility; executive function; network dynamics; network interactions; task representation.

\section{Significance Statement}

Cognitive control supports a variety of behaviors requiring flexible cognition, such as rapidly switching between tasks. Furthermore, cognitive control is negatively impacted in a variety of mental illnesses. We used tools from network science to characterize the implementation of cognitive control by large-scale brain systems. This revealed that two systems, the frontoparietal (FPN) and cingulo-opercular (CON) networks, have distinct but complementary roles in controlling global network reconfigurations. The FPN exhibited properties of a flexible coordinator (orchestrating task changes), while CON acted as a flexible switcher (switching specific regions to other systems to lend processing resources). These findings reveal an underlying distinction in cognitive processes that may be applicable to clinical, educational, and machine learning work targeting cognitive flexibility.

\footnotetext{
Received Oct. 27, 2019; revised June 28, 2020; accepted June 30, 2020

Author contributions: C.V.C. and M.W.C. designed research; C.V.C., T.I., D.S., and M.W.C. performed research; C.V.C., T.I., D.S., D.S.B., and M.W.C. contributed unpublished reagents/analytic tools; C.V.C. analyzed data; C.V.C. wrote the paper.

The authors declare no competing financial interests.

This work was supported by National Institutes of Health Grants K99-R00 MH096901 and R01 MH109520. The authors acknowledge the Office of Advanced Research Computing (OARC) at Rutgers, The State University of New Jersey for providing access to the Amarel cluster and associated research computing resources. The authors thank their colleagues at the Cole Neurocognition Lab and the Rutgers University Brain Imaging Center (RUBIC) for their expertise and diligent efforts in data collection, as well as offering words of wisdom and overall support.

Correspondence should be addressed to Carrisa V. Cocuzza at carrisacocuzza@gmail.com.

https://doi.org/10.1523/JNEUROSCI.2559-19.2020

Copyright $\odot 2020$ the authors
}

\section{Introduction}

Theories of cognitive control, processes supporting goal-directed cognition and behavior, suggest the need for flexibly reconfigurable neural systems to support controlled processing (Desimone and Duncan, 1995; Miller and Cohen, 2001; Schneider and Chein, 2003; Cole et al., 2013b). In order for an individual's goals to be implemented, goal-relevant information must be appropriately represented across large-scale neural systems, or networks. Importantly, goals and goal-relevant information are subject to change over time (such as sensorimotor information that corresponds to changing task conditions). Processing these dynamic 


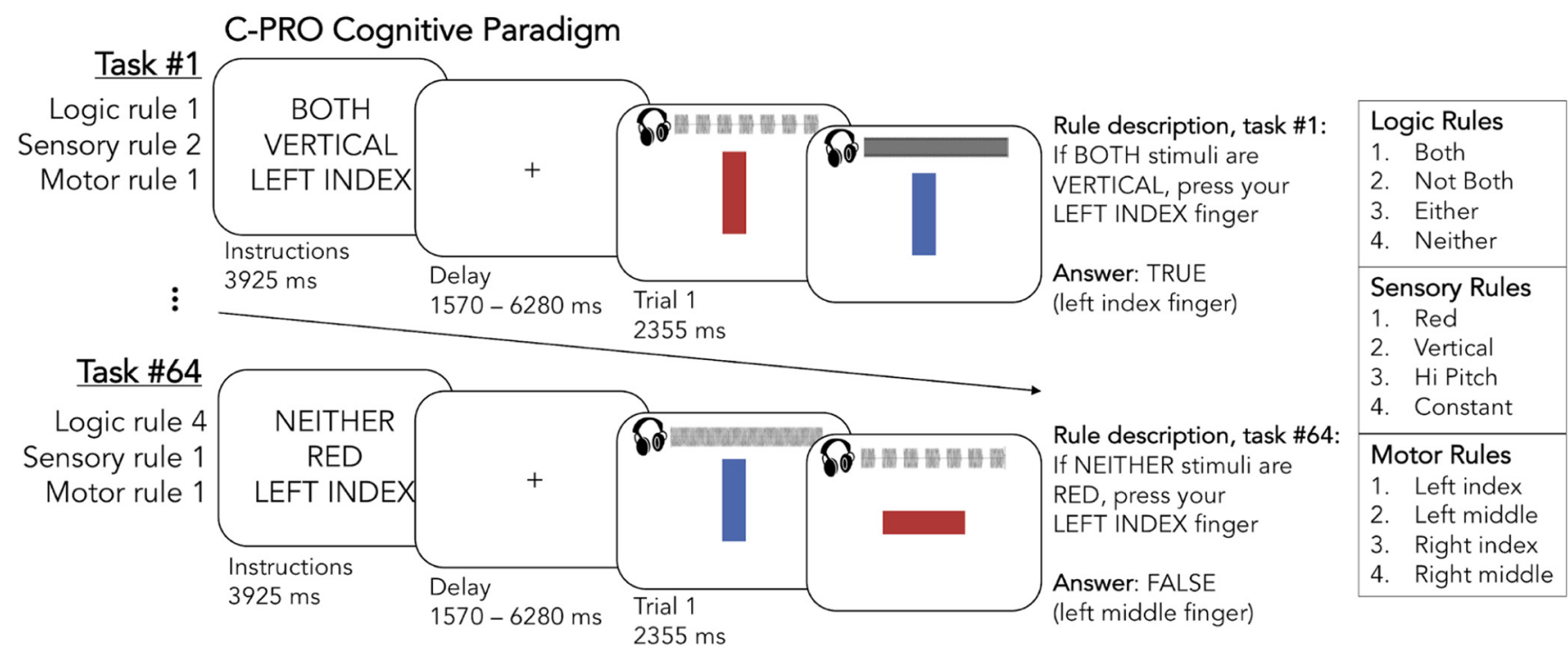

Figure 1. The C-PRO cognitive paradigm. First, an instruction screen presented the rules for a given task $(3925 \mathrm{~ms})$. Participants next applied these rules to pairs of consecutively presented audiovisual stimuli (auditory waveforms are visually depicted here but were only presented audibly to participants). Two example task-rule sets are depicted, as well as how participants were trained to interpret the rules (e.g., rule descriptions on the right-most portion of the figure; see Materials and Methods for details). The 12 possible rules are listed on the right.

changes must be guided among neural systems that represent goal-relevant information. Cognitive control networks are proposed to enact this guidance via network interactions that are flexible with respect to the current task context (Waskom et al., 2014). Thus, we focus here on the role of large-scale network dynamics as task goals are updated across 64 systematically-related task contexts (Fig. 1).

The theoretical insight that large-scale network interactions are essential to cognitive control evolved over the last several decades, beginning with empirical observations (Fuster et al., 1985), which then led to the biased competition theory (Desimone et al., 1990; Desimone and Duncan, 1995). This theory focused on lateral prefrontal cortex influencing the visual system by biasing its competition for attentional resources toward goal-relevant representations. Building on the biased competition theory, the guided activation theory generalized this prefrontal network mechanism to all task domains. This theory proposed a general role for top-down prefrontal influences in accomplishing task goals (Miller and Cohen, 2001). More recently, the flexible hub theory generalized the guided activation theory beyond prefrontal cortex to the entire frontoparietal network (FPN) and formalized the importance of cross-network, global connectivity changes in implementing cognitive control (Cole et al., 2013b). The present study builds on this work to further verify and expand the flexible hub theory.

Simultaneous with these advances in theory have been observations of a second major neural system supporting cognitive control: the cingulo-opercular network (CON). Like the FPN, the CON is active as a function of cognitive control demands across a wide variety of tasks (Dosenbach et al., 2006; Yeo et al., 2015; Crittenden et al., 2016). However, CON and FPN are not equally active for all task conditions (Dosenbach et al., 2006; Yeo et al., 2015), and they maintain distinct functional network architectures in terms of resting-state functional connectivity (rsFC; Dosenbach et al., 2007; Power et al., 2011; Ji et al., 2019) and task-state FC (tFC; Cole et al., 2014; Crittenden et al., 2016). Moreover, the specific functional contributions of $\mathrm{CON}$ regions have not been fully established, with some studies suggesting that CON regions specify overall task set modes of processing (Dosenbach et al., 2007; Sadaghiani and D'Esposito, 2015) and others emphasizing the CON's role in reactive (phasic) attention (Seeley et al., 2007) and, relatedly, conflict processing (Botvinick, 2007; Cole et al., 2009; Braem et al., 2019). Ultimately, unlike the FPN, the relationship between the CON and the flexible hub theory (and the theories it builds on) remains unclear.

The present study builds on our prior work demonstrating flexible hub properties in FPN regions (Cole et al., 2013b), expanding on the characterization of these FPN network mechanisms, while also investigating CON network mechanisms. We previously found that FPN's global tFC patterns flexibly updated according to task demands more than any other network, including CON (Cole et al., 2013b). However, given that large-scale network dynamics are central to cognitive control, and given that both the CON and FPN contain hubs (Power et al., 2011; Ito et al., 2017), we hypothesized that CON reflects flexible hub properties in addition to FPN. Unlike FPN's continuous goalcoordinating role, we expected $\mathrm{CON}$ to exhibit a more discrete network switching mechanism, reflecting its proposed role in specifying overall task-set modes of processing (Dosenbach et al., 2007; Sadaghiani and D’Esposito, 2015). Consistent with this, we found that FPN regions act as "flexible coordinators" and CON regions as "flexible switchers," providing separate but complementary network mechanisms in support of cognitive control (Fig. 2).

\section{Materials and Methods}

\section{Participants}

Right-handed, healthy adult participants $(N=106)$ were recruited from Rutgers University and the surrounding Newark, New Jersey community. Six participants were excluded from analyses because of technical errors, leaving a total sample size of $N=100$ (Tables 1, 2 detail demographic characteristics). To improve replicability, we used a split-sample validation approach (Anderson and Magruder, 2017) with a random subset of $n=50$ comprising a discovery dataset (Table 1 ), and the remaining $n=50$ comprising a replication dataset (Table 2). All participants provided informed consent in accordance with protocols approved by the Institutional Review Board of Rutgers University-Newark. Each participant provided or completed the following: (1) demographic information and intake survey questions; (2) the National Institutes of Health Cognition Toolbox (Gershon et al., 2013), including a neuropsychological 


\section{A $\quad$ FPN regions act as "flexible coordinators"}

across task states: high global variability (high within, high between), low partition deviation (low within, $\bar{x}$ between)

Intrinsic State

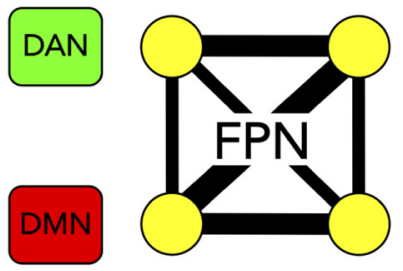

Task State 1

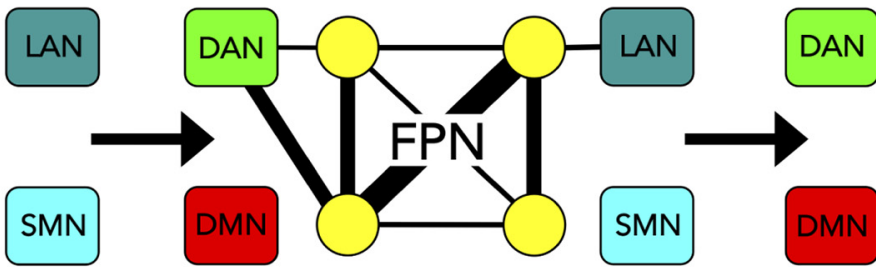

Task State 2

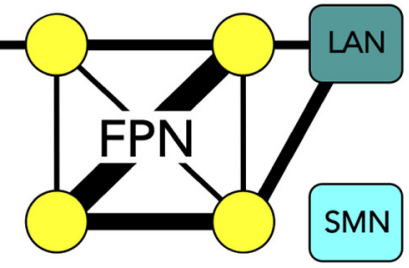

\section{B}

CON regions act as "flexible switchers"

across task states: low global variability ( $\bar{x}$ within, low between), high partition deviation (high within , $\bar{x}$ between)

\section{Intrinsic State}
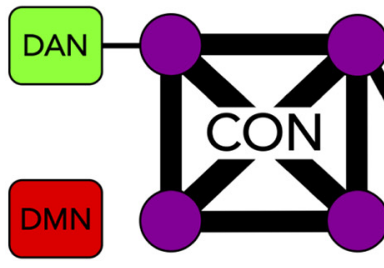

\section{Task State 1}
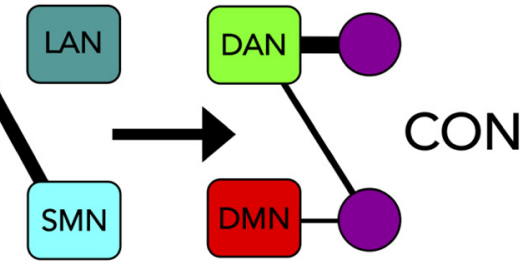
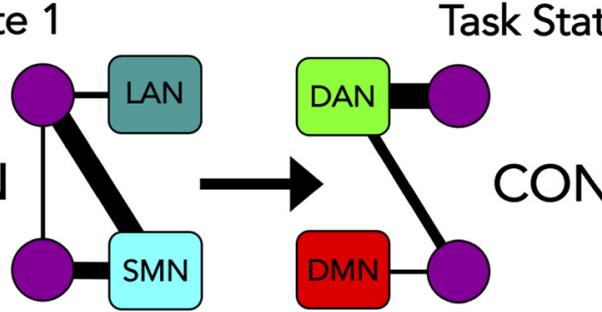

Task State 2

Figure 2. Schematic depictions of cognitive control network functional properties (details on network measures, see Materials and Methods and Results). In each panel, a "toy" version of the control network is prominently depicted in the center (with a reduced number of regions, or nodes, and simplified within-network connections), and out-of-network exemplars are depicted as truncated and surrounding the control network of interest (DAN: dorsal attention network, LAN: language network, DMN: default mode network, and SMN: somatomotor network). Each of these surrounding networks also contains within-network regions and connections, but these were not depicted here for simplicity. $\boldsymbol{A}$, Regions in the FPN acted as flexible coordinators. This entailed high GVC and low partition deviation across task states. From example task state one to task state two, FPN regions maintained their within-network connectivity (low deviation) and out-of-network connectivity changes were variable across states (high GVC; for details, see Results). $\boldsymbol{B}$, Regions in the CON acted as flexible switchers. This entailed low global variability and high partition deviation. From example task state one to task state two, CON regions dropped their within-network connectivity (high deviation) and out-of-network connectivity changes were consistent across states (low GVC; for details, see Results).

Table 1. Demographic characteristics of the discovery dataset $(n=50)$

\begin{tabular}{|c|c|c|c|c|c|c|c|c|c|}
\hline & \multicolumn{4}{|c|}{ Male $(n=19)$} & \multicolumn{4}{|c|}{ Female $(n=31)$} & \multirow[b]{2}{*}{ Test (male vs female) ${ }^{* *}$} \\
\hline & $n$ & $\%$ & Center* & $S D( \pm)$ & $n$ & $\%$ & Center* & $S D( \pm)$ & \\
\hline Age (years) & & & 21.2 & 2.9 & & & 19.9 & 1.6 & $t_{(48)}=1.71, p=0.10$ \\
\hline $25-34$ & 2 & 10.5 & & & 0 & 0 & & & \\
\hline $35-44$ & 0 & 0 & & & 0 & 0 & & & \\
\hline Ethnicity/race & & & White & $\mathrm{n} / \mathrm{a}$ & & & Black & $\mathrm{n} / \mathrm{a}$ & $\chi^{2}(5, N=50)=6.29, p=0.18$ \\
\hline Black or African American & 3 & 15.8 & & & 12 & 38.7 & & & \\
\hline Hispanic or Latino & 4 & 21.1 & & & 2 & 6.5 & & & \\
\hline Native Hawaiian or Pacific Islander & 0 & 0 & & & 0 & 0 & & & \\
\hline White or white & 7 & 36.8 & & & 6 & 19.4 & & & \\
\hline Other & 0 & 0 & & & 1 & 3.2 & & & \\
\hline Education & & & Student & $\mathrm{n} / \mathrm{a}$ & & & Student & $\mathrm{n} / \mathrm{a}$ & $\chi^{2}(2, N=50)=1.27, p=0.26$ \\
\hline
\end{tabular}

There were no significant differences between identified genders on the distributions of age, ethnicity, or education (right column).

*The measure of center used for the age variable was the mean, and for categorical variables of ethnicity/race and education it was the mode. For the education variable, student refers to "some college."

**Hypothesis testing of significant differences between males and females. Age: two-sample $t$ test adjusted for unequal sample sizes. Ethnicity/race and education: a $\chi^{2}$ test of independence.

battery; (3) behavioral training on the C-PRO task (outside the scanner); (4) resting-state fMRI; and (5) C-PRO task fMRI. The subsets of data assessed herein included (1), (4), and (5) (for assessment of other variables, see Schultz et al., 2019). As listed in the right-most columns of Tables 1,2 , there were no significant differences between identified genders on the distributions of age, ethnicity, or education.
Concrete permuted rule operations (C-PRO) paradigm

The C-PRO paradigm was designed to involve rapid instructed task learning (RITL) through compositionally combining various task rules (Cole et al., 2010, 2013a; Ito et al., 2017). This further provided a high demand on cognitive control across all C-PRO task states. We used a modified version of the PRO paradigm from Cole et al. (2010), which 
Table 2. Demographic characteristics of the replication dataset $(n=50)$

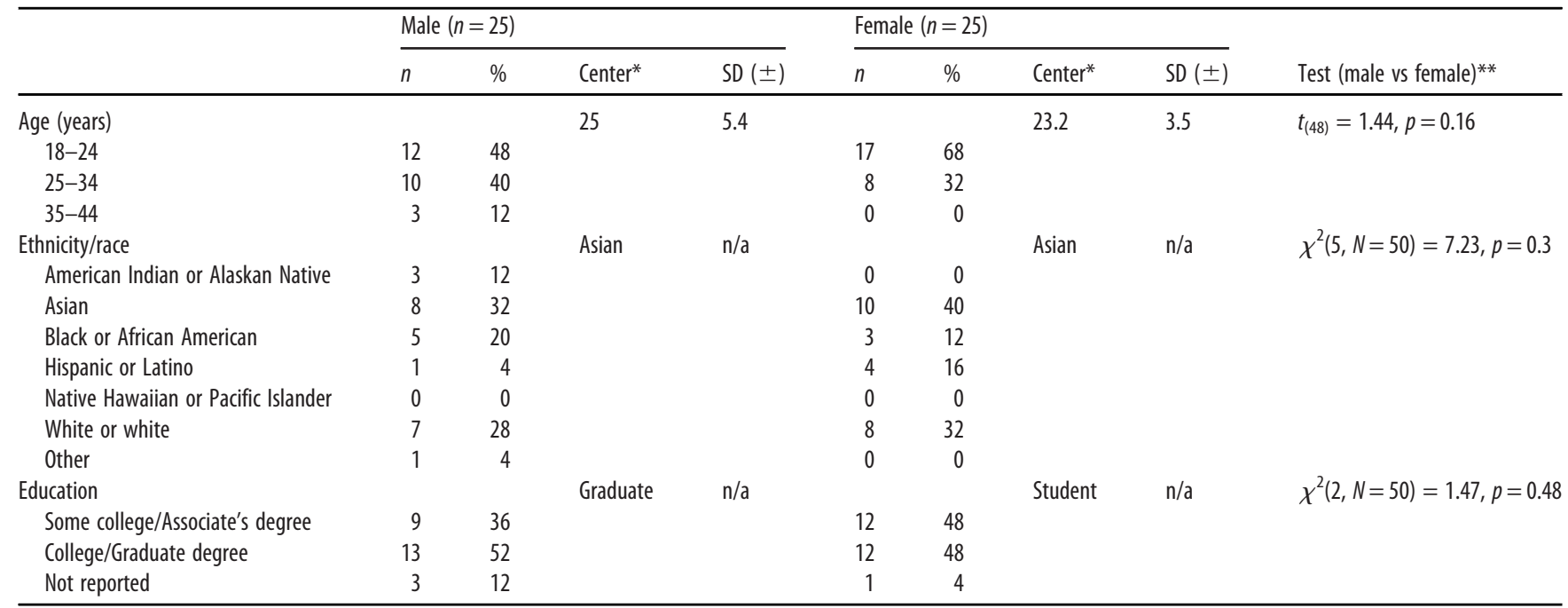

All table features are the same as in Table 1. Note that there were no significant differences between identified genders on the distributions of age, ethnicity, or education (right column).

was previously introduced by Ito et al. (2017; Fig. 1). This paradigm permutes rules across three domains: four logic rules (both, not both, either, and neither), four sensory rules (red color, vertical orientation, high pitch sound, and constant tone), and four motor rules (left index, right index, left middle, and right middle fingers). This amounts to 12 rule sets represented 16 times across 64 unique task states. The software for presenting the task was E-Prime version 2.0.10.353 (Schneider et al., 2002).

In each task state, an initial instruction screen was presented for $3925 \mathrm{~ms}$ for participants to memorize a given permuted rule set (Fig. 1). This was followed by a jittered delay (1570-6280 ms, randomized from a uniform distribution), then three trials of paired audiovisual stimuli for participants to adjudicate based on the given rule set $(2355 \mathrm{~ms}$ each trial; inter-trial interval of $1570 \mathrm{~ms}$ ). Another jittered delay occurred at the end of each task state (7850-12,560 ms, randomized), which was immediately followed by the next permuted rule set instruction screen. An example instruction screen (Fig. 1; task state one) read: "BOTH, VERTICAL, LEFT INDEX," indicating: "If both stimuli are vertical, press your left index finger." In each of the three trials that followed, participants judged whether both paired stimuli were vertically oriented, and either pressed the left index finger button to indicate "true" or the left middle finger button to indicate "false" (a judgment of false was always the same hand but opposite finger). Importantly, stimuli were always presented with auditory and visual features concurrently. Thus, focusing on the sensory rule given by the instructions was paramount (i.e., "VERTICAL" indicated that one should ignore auditory information, color information, and only focus on line orientation). Additionally, participants were required to remember and apply conditional logic and nontrivial motor commands each trial. Altogether this multitask behavioral paradigm is condition-rich and necessitates ongoing cognitive control.

Each participant completed a training session outside the scanner and a testing session within the scanner (task-state fMRI) 30 min later. During the training session participants equally practiced four rule sets that contained all 12 rules. This practice set was counterbalanced among participants and supplementary instruction was provided for training purposes (e.g., use the same hand but opposite finger to indicate false). Task fMRI scans were performed in eight runs, altogether containing 64 task state miniblocks, twice over (e.g., 128 task miniblocks), with each block composed of a permuted rule set (Fig. 1). Each task fMRI run was $\sim 8 \mathrm{~min}$ in duration, and identical miniblocks were never presented consecutively. Overall, mean performance was $83.47 \%$ correct $(S D=9.00 \%)$. There was no significant difference in performance (percent correct) between males $(\mathrm{M}=83.65 \%, \mathrm{SD}=10.44 \%)$ and females $(\mathrm{M}=83.33 \%, \mathrm{SD}=7.80 \%) ; t_{(74.71)}=0.17, p=0.87$.

\section{Experimental design and statistical analysis}

Participants were randomly allocated to either a discovery dataset $(n=50)$ or replication dataset $(n=50$; Tables 1,2 , respectively). The replication dataset was not analyzed until after analyses of the discovery dataset were complete. Analyses of replication data were identical to analyses of discovery data (using the same code, including all chosen parameters), and additionally included measures of similarity between replication and discovery results to quantify expected generalizability (Anderson and Magruder, 2017).

Whenever multiple comparisons were addressed, we used the Max-T nonparametric permutation testing approach $(10,000$ permutations unless otherwise specified) with maxima-derived $95 \%$ confidence intervals for statistical hypothesis testing against zero (Blair and Karniski, 1993; Nichols and Holmes, 2002). To analyze the similarity of two correlation (weighted adjacency) matrices, we used the Mantel permutation test, which performs a Pearson's correlation across the upper triangles (off-diagonal) of the matrices (Mantel, 1967; Glerean et al., 2016). The Mantel test is more conservative than a standard comparison between connectivity matrices because it takes into account the fact that observations in distance/similarity matrices are not independent (an assumption of both parametric and standard non-parametric tests). In each Mantel analysis, we again used nonparametric permutation procedures to derive statistics that make minimal assumptions about probability distribution (10,000 permutations unless otherwise specified). Henceforth we will describe these matrix similarity statistics as Mantel- $r$.

\section{MRI parameters}

All MRI data were collected at the Rutgers University Brain Imaging Center (RUBIC). When possible, the best practices suggested by the Human Connectome Project preprocessing pipelines were followed (Glasser et al., 2013). A 3T, 32-channel head coil within a Siemens Trio scanner was used to obtain multiband, whole-brain, and echoplanar imaging (EPI). The repetition time (TR) was $785 \mathrm{~ms}$; the echo time (TE) was $34.8 \mathrm{~ms}$; the flip angle was $55^{\circ}$; the bandwidth was $1924 \mathrm{~Hz} / \mathrm{Px}$; the in-plane field-of-view (FoV) read was $208 \mathrm{~mm}$; 72 slices; $2.0 \mathrm{~mm}$ isotropic voxels; and the multiband acceleration factor was 8 . Whole-brain and high-resolution T1-weighted and T2-weighted anatomic scans were also acquired, with an isotropic voxel resolution of $0.8 \mathrm{~mm}$. Spin echo field maps were obtained in both the anterior-posterior and posterioranterior directions. Resting-state fMRI scans were $14 \mathrm{~min}$ in duration, amounting to 1070 TRs. Each task (i.e., C-PRO) fMRI run was $\sim 8 \mathrm{~min}$ in duration, adding up to $\sim 1 \mathrm{~h}$ in the scanner for the task session (36 TRs per task miniblock; 4608 TRs altogether).

\section{fMRI preprocessing}

The open-source Human Connectome Project minimal preprocessing pipeline (Glasser et al., 2013), version 3.5.0, was applied to all neuroimaging data. This included: anatomic reconstruction and segmentation; 
EPI reconstruction, segmentation, and spatial normalization to a standard template; intensity normalization; and motion correction. The resulting data were in CIFTI 64k-vertex grayordinate space, and all subsequent analyses were performed in MATLAB R2014b (The MathWorks). Following minimal preprocessing, vertices were parcellated into 360 cortical regions (180 per hemisphere) per the Glasser et al. (2016) atlas. To parcellate each of these regions, we calculated the average time series of enclosed vertices.

Next, we performed nuisance regression on parcellated resting-state and task-state data using six motion parameters plus their derivatives (totaling 12 motion parameters), and volumetrically-extracted ventricle and white matter time series (via FreeSurfer; http://surfer.nmr.mgh. harvard.edu/), plus their first derivatives (16 regressors overall). Note that global signal was not removed because of evidence that it can artificially introduce negative relationships (Murphy et al., 2009). Task time series were further processed to account for confounding effects introduced by simultaneous sensory inputs (e.g., left and right primary visual area, V1) and their downstream effects by fitting a general linear model (GLM) to task activity estimated by a finite impulse response (FIR) function. This removal of cross-event mean task-locked activity has been shown to reduce task-evoked correlation false positives while retaining most $(\sim 90 \%)$ of the correlated variance between fMRI time series and without inflating false negatives (Cole et al., 2019). In the task GLM, each task run was separately demeaned, and drift was accounted for with a per-run linear trend.

\section{FC estimation}

FC was estimated for parcellated (region-wise), pre-processed data, per participant and per state (one resting state and 64 C-PRO task states). Across the whole cortex, we used Fisher's Z-transformed Pearson correlation coefficients to compute interregional relationships of blood oxygen level-dependent (BOLD) time series, resulting in $360 \times 360$ connectivity matrices. Given the complex nature of subsequent analyses (i.e., network metrics), we chose this method of FC estimation for simplicity and wide-reaching comprehension. In the present study, connectivity estimates tended to decrease from rest to task, a finding that has been observed across numerous prior studies (that used various model species and neural recording methods; Cohen and Maunsell, 2009; He, 2013; Cole et al., 2014; Ponce-Alvarez et al., 2015) and has well-founded neural mechanisms (Ito et al., 2019).

We chose to use FIR regression to remove cross-block mean taskevoked activations before Pearson correlation estimation (sometimes termed "background connectivity," as in Norman-Haignere et al., 2012) based on recent results demonstrating that this approach was better able to remove confounding effects of task-evoked activity than alternative approaches, such as psychophysiological interactions (PPIs; Cole et al., 2019). Our prior global variability coefficient (GVC; see below, Network metrics) results were based on generalized PPI connectivity estimates (Cole et al., 2013b), such that the present results provided improved testing of the flexible hub theory.

\section{Network partition}

We applied the cortical portion of the Cole-Anticevic brain-wide network partition (CAB-NP; Ji et al., 2019; Fig. 3), which was based on publicly available Human Connectome Project data. The CAB-NP was based on resting-state fMRI data across the whole brain, and used the Louvain community detection algorithm to assign parcellated cortical regions (Glasser et al., 2016) into 12 functional networks. The CAB-NP corroborated features of well-known cortical partitions (Power et al., 2011; Yeo et al., 2011; Gordon et al., 2016), yet found novel but robust networks. The CAB-NP was implemented for all analyses except network flexibility (NF), which requires the application of community detection (Louvain Q-modularity; see below, NF).

Given that our novel network metric (see below, Network partition deviation) quantifies network affiliation changes from an intrinsic partition, it was important (to avoid inflated deviation estimates) to ensure that the intrinsic partition was applicable to the present group of subjects. We first partitioned resting-state data by sorting regional FC estimates per the 12 CAB-NP network indices. We then found the maximum FC estimate (i.e., the intrinsic "preference") for each region (per participant) and tested whether its location was equivalent to the CAB-NP. If this index was different from the CAB-NP in over $50 \%$ of participants, we reassigned that region to its empirically-derived preference. We henceforth used this empirically-adjusted CAB-NP to sort task-state data into networks (Fig. 3C,D).

In select analyses, we probed the similarity of two partitions. To accomplish this, we used the Jaccard index, which is a standard measure of similarity from set theory. For example, the Jaccard index was used to assess the similarity of the empirically-adjusted resting-state partitions of the discovery and replication datasets. We used the MATLAB jaccard function, which used the "intersection over union" formula on label vectors $\mathrm{A}$ and $\mathrm{B}$, with the following equation:

$$
\operatorname{jaccard}(A, B)=|\operatorname{intersection}(A, B)| /|\operatorname{union}(A, B)| \text {. }
$$

Per state, the intersection equaled the number of true positives (i.e., overlap of two partitions), and the union was the number of true positives summed with the number of false positives and false negatives.

\section{Network metrics}

Interregional connectivity was probed by three network metrics for state-based reconfiguration properties (Medaglia et al., 2015): (1) GVC (Cole et al., 2013b) and, relatedly, between-network variability coefficient (BVC; novel but related to Ito et al., 2017); (2) NF (Bassett et al., 2011, 2013a); and (3) network partition deviation (deviation; novel). Network metrics were computed across states and averaged across regions that compose a given network, per participant. In analyses that used standardized metrics (i.e., $z$ scores), standardizations were performed before network averages and standard errors were computed. Figure 4 illustrates the algorithms of these metrics schematically. Table 3 summarizes the primary characteristics of these metrics, including formulae, interpretations, parameter-space considerations, and reliance on a predefined network partition. A predefined network partition is sometimes called a "hard partition," and refers to the use of a predefined network or community assignment structure, such that each parcellated region is indexed into the partition a priori (Sporns and Betzel, 2016).

\section{GVC}

GVC was originally developed by Cole et al. (2013b) and characterizes changing patterns of connectivity across task states by measuring the variability of interregional connectivity (Fig. 4A; Table 3). Thus, GVC treats spatial changes in connectivity, across states, as continuous. No parameters are required by the user and a predefined network partition is not necessary (aside from regional parcellation, as in the present study; Table 3).

In Cole et al. (2013b), FPN connections exhibited the highest GVC compared with all other networks. In that study, the FPN also maintained connectivity patterns that could decode task information (using an earlier version of the C-PRO paradigm). Further, FPN connectivity was found to vary systematically with similarity of C-PRO task states. Taken together this suggested that (1) FPN regions exert adaptive task control as flexible hubs, and (2) GVC results were not driven by noise. We replicated these findings and extended the analysis to CON connections. In brief, the $64 \mathrm{C}$-PRO task states have zero to two overlapping rules (Fig. 1). For example, one task's rules included both, high pitch, and left middle, and another included both, red, and left middle. These example tasks had two overlapping rules (both and left middle). We created a $64 \times 64$ similarity matrix to quantify these overlap sets, and quantified the Spearman's Rho for FPN and CON connections for those sets. Next, we restricted the same analysis by only including FPN and CON regions with the highest GVC, in the following increments: top $10 \%, 8 \%$, $6 \%, 4 \%$, and $2 \%$. This addresses whether highly variable connectivity (as measured by GVC) relates systematically to task context (Cole et al., 2013b).

\section{$B V C$}

BVC was inspired by Cole et al. (2013b) and is related to between-network global connectivity in Ito et al. (2017). BVC is equivalent to GVC, 


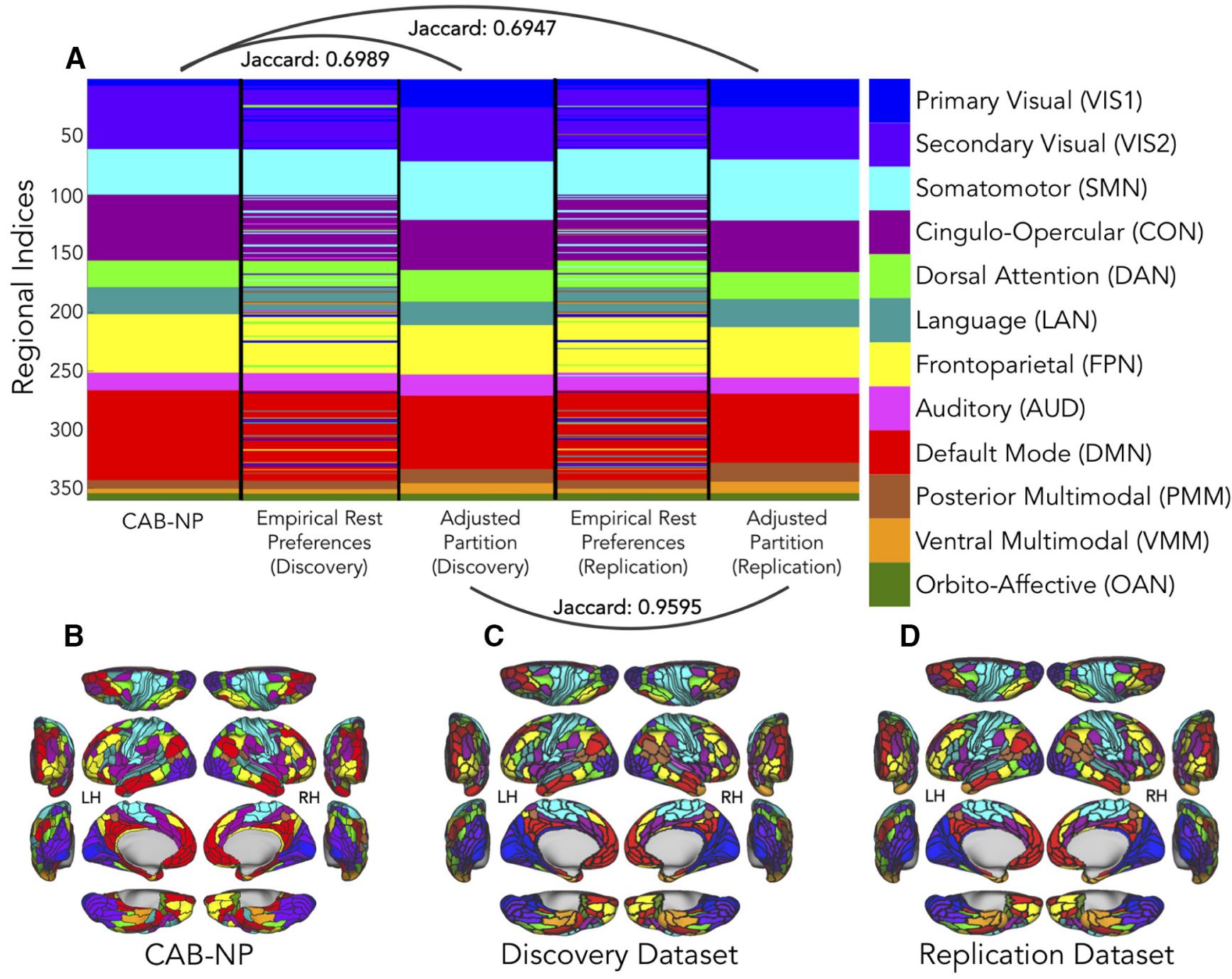

Figure 3. The CAB-NP adjusted by empirical rsFC, for both the discovery and replication datasets. Given that the novel metric, deviation (see below, Network partition deviation), quantified network affiliation changes from an intrinsic partition, empirical adjustments to the CAB-NP ensured that partition was applicable to the present group of subjects and deviation scores were not inflated. We utilized this adjusted partition for all other (applicable) network measures for consistency with deviation. $\boldsymbol{A}$, Regional ( $y$-axis; Glasser et al., 2016 parcels) assignments are color-coded according to the CAB-NP (rightmost scale). The CAB-NP column depicts the original resting-state network partition by Ji et al. (2019). The empirically-derived rest preferences are shown, unordered, for both the discovery and replication datasets, as well as their ordered counterparts (i.e., "adjusted partitions"). These adjusted partitions were used for all analyses. $\boldsymbol{B}$, CAB-NP by Ji et al. (2019) projected onto brain regions. C, The empirically-adjusted CAB-NP for the discovery dataset projected onto brain regions. The Jaccard similarity coefficient between the CAB-NP and the empirically-adjusted discovery set partition was 0.6989 . D, The empirically-adjusted CAB-NP for the replication dataset projected onto brain regions. The Jaccard similarity coefficient between discovery and replication partitions was 0.9595 , suggesting the partition method used herein will have high external validity. The Jaccard similarity coefficient between the CAB-NP and the empirically-adjusted replication set partition was 0.6947. This suggests a relatively high similarity between each of the empirically-adjusted partitions (discovery and replication) and the CAB-NP. The least similarity was observed in the VIS1, which was expanded to include CAB-NP secondary visual and dorsal attention regions in the empirical adjustments.

except within-network connectivity estimates are withheld from the computation of SD (Fig. 4A). This change from GVC accounts for the potential confound that within-network connections might confer on results. In Cole et al. (2013b), FPN had the highest participation coefficient compared with all other networks, suggesting that FPN regions maintain many between-network connector hubs. BVC simply quantifies this in a manner closer to GVC. BVC (unlike GVC) required the use of a predefined network partition to define the regional bounds of each network. All other specifications of BVC are identical to GVC (Table 3).

$N F$

NF was originally developed by Bassett et al. (2011, 2013a,b) to quantify how often (i.e., for how many tasks) a region changes its network "allegiance" and standardizes this by all possible changes. NF is conceptually related to GVC because both metrics quantify large-scale changes in functional network configurations. In the present study, we specifically tested whether NF and GVC estimate comparable aspects of network configuration. NF characterizes the spatiotemporal dynamics related to task-state time series by quantifying temporal variability in network partition solutions. These network partitions are determined by an optimized quality function for community detection termed multilayer modularity (also termed multislice or multiplex in some studies; Louvain Q-modularity; Mucha et al., 2010). Thus, NF does not use a predefined network partition, but instead requires community detection to be applied per dataset. Required parameters $(\gamma, \omega)$ could be used to tune the degree to which connections were treated as discrete versus continuous in space ( $\gamma$ is the spatial resolution parameter) and/or time ( $\omega$ is the temporal resolution, or coupling, parameter). In the present study, we swept a parameter space of $\gamma$ and $\omega$, ranged around their prototypical values (Bassett et al., 2013a; Braun et al., 2015; Chen et al., 2015; Amelio and Tagarelli, 2017). We swept the modularity function's parameter space by ranging $\omega$ from 0 to 2.0 in steps of 0.2 ; and $\gamma$ from 0 to 5.0 in steps of 0.5 . For both free parameters, 0 is the lower limit. An upper limit of 2.0 for $\omega$ was based on prior observations that task states 
A
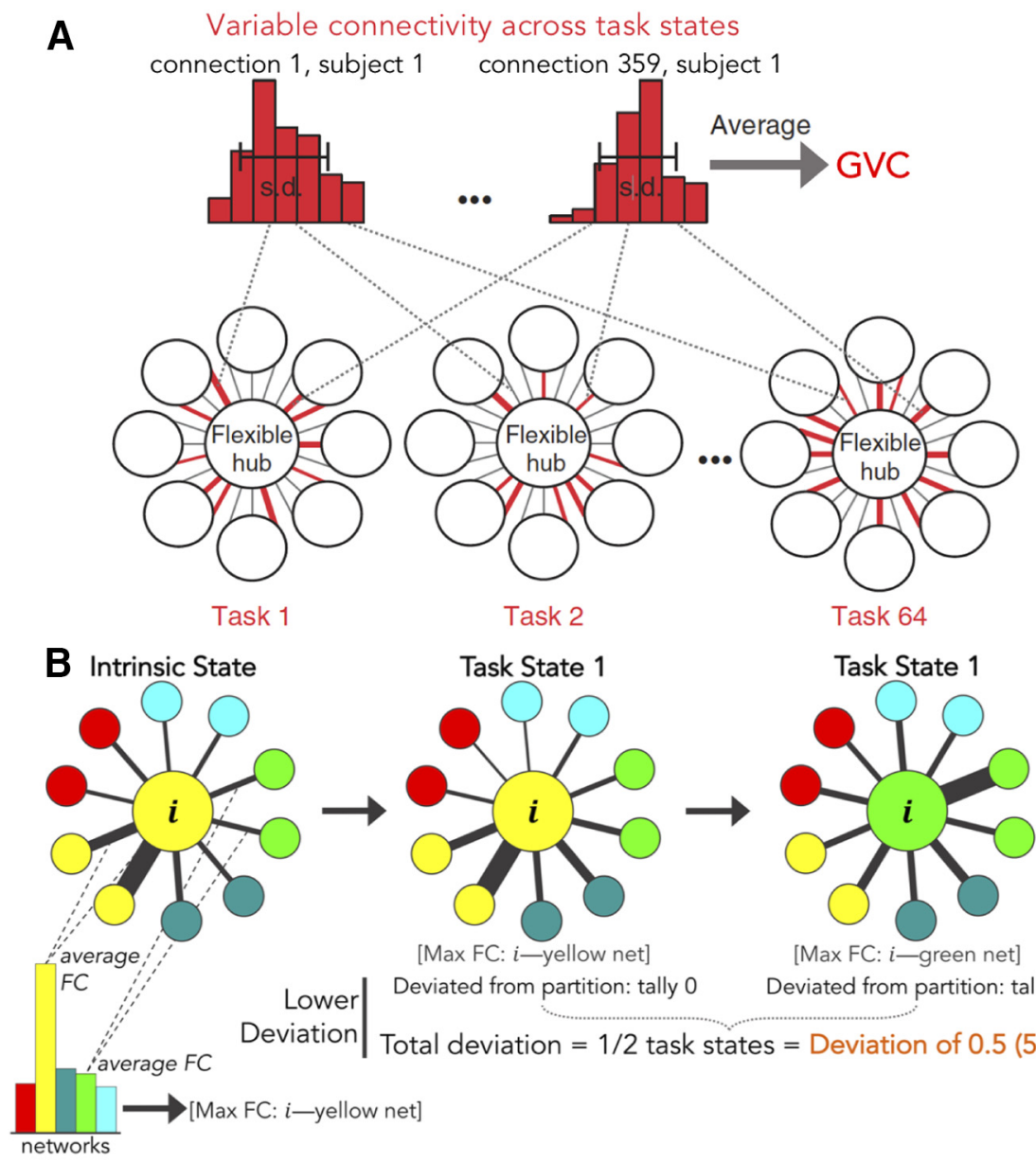

Task State 1

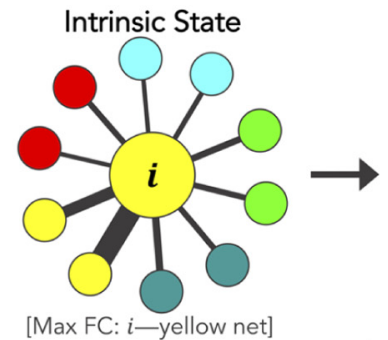

Task State 1
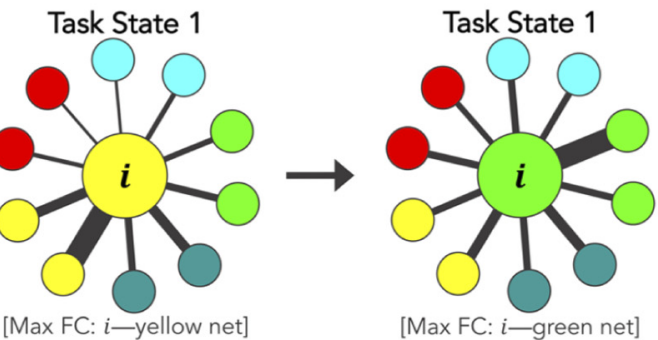

[Max FC: $i$-green net]

eviated from partition: tally $0 \quad$ Deviated from partition: tally 1

Total deviation $=1 / 2$ task states $=$ Deviation of $0.5(50 \%)$

$$
\begin{array}{r}
\text { Higher } \\
\text { Deviation }
\end{array} \mid \begin{aligned}
\text { Deviated from partition: tally } 1 \quad \text { Deviated from partition: tally } 2 \\
\text { Total deviation }=2 / 2 \text { task states }=\text { Deviation of } 1(100 \%)
\end{aligned}
$$

Figure 4. Schematic depictions of network metric algorithms. A, GVC, reproduced with permission (Cole et al., 2013b). BVC is measured equivalently, except within-network connections are withheld. $\boldsymbol{B}$, Network partition deviation. Per region (large yellow example node labeled " $\mathrm{i}$ "): each of its 359 connectivity estimates were averaged according to their CAB-NP (see Materials and Methods; Fig. 3) networks (bar graph in top example), resulting in 12 FC estimates per region. Network "preferences" (network location of maximum FC estimate; thickest lines) were tallied across task states. How often a given region deviated from its predefined partition (intrinsic state) was computed (tally/total number of tasks). Lower deviation: the example region deviated in one out of two hypothetical task states ( $50 \%$ deviation $=$ deviation of 0.5$)$. Higher deviation: the example region deviated in two out of two hypothetical states $(100 \%$ deviation $=$ deviation of 1). The colored nodes encircling the example region represent example regions from example networks, and black lines of variable width represent FC estimates (edge weights).

tend to merge into one large state at higher coupling values (Bassett et al., 2013a). At the upper limit of $\gamma(5.0)$, spatial resolution becomes acute and each region develops its own network. Additionally, the temporal dynamics conferred by $\omega$ are no longer available at the upper limit of $\gamma$ (Chen et al., 2015; Amelio and Tagarelli, 2017). This sweep yielded an 11 by 11 matrix of NF estimates, per region (and per participant). We compared regional NF estimates to regional GVC estimates (both metrics were standardized, and Spearman's rank-order correlation quantified similarity in these measures across participants), to assess the point in the parameter-space wherein NF and GVC overlap most. These comparisons were performed for both the networkmean and regional-mean vectors. Briefly, we found that NF and GVC characterized shared aspects of network configurations in a specific sector of NF's parameter-space (see Results). This motivated the development of a novel metric (see below, Network partition deviation) that was less linked to chosen parameters. The remaining results are based on GVC and this novel metric (such as the cognitive cartographies; see Results). Importantly, however, there are future research questions that may be better addressed by NF.

\section{Network partition deviation}

To reconcile divergent principles and results of GVC and NF (see Results), we created a novel metric termed network partition deviation (or just "deviation"). The primary goal of developing deviation was to quantify network reconfiguration in a highly principled manner. This involved a principled definition for what it means for a region to 
Table 3. Summary of the network metrics for cognitive control properties across states

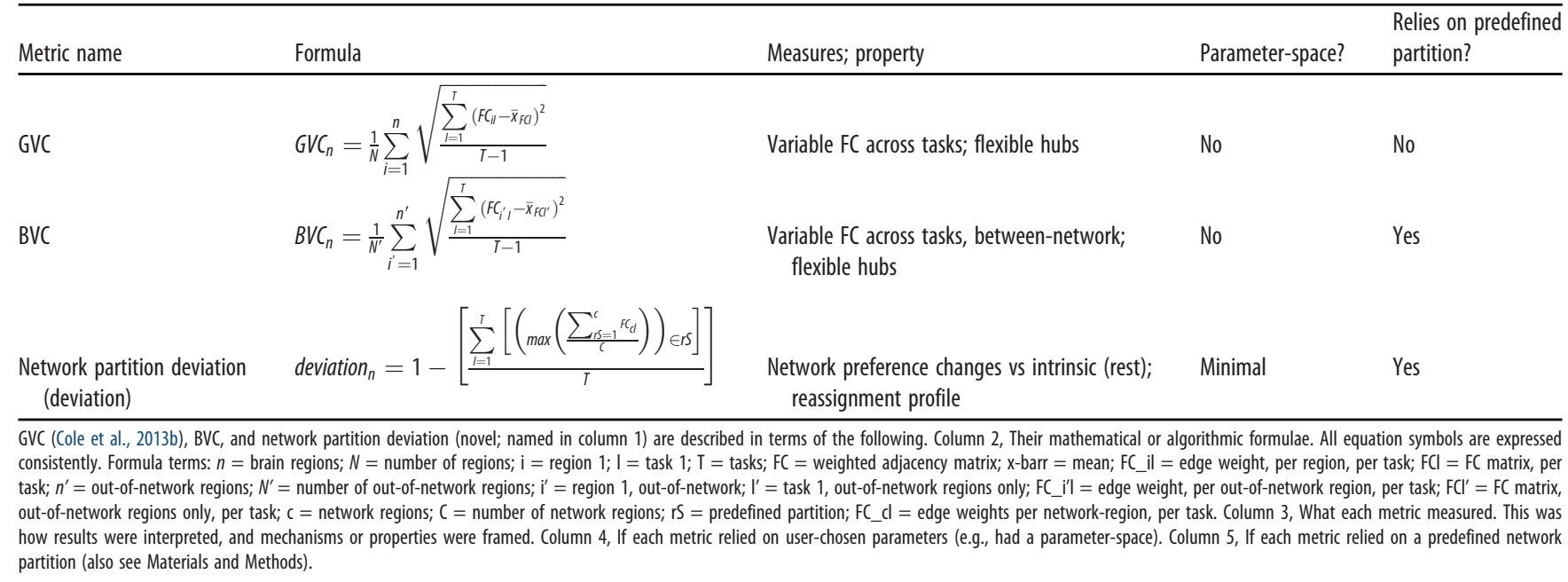

"reconfigure": a change in the network community that a given region is most connected with (i.e., the network with the highest mean connectivity). Deviation was the percent of task states (more generally, the relative frequency across time), in which a given region's "preference" deviated from the predefined partition. To quantify this, deviation enumerated network reassignments from a predefined partition across task states (Table 3; Fig. 4B). Per task state and per region, connectivity estimates (across the other 359 regions) were searched for the maximum value. The network location of this maximum (relative to the predefined partition) was indexed as the network assignment preference for that given state. To illustrate how network reassignment was computed, we generated a video of the regional network preferences across task states, projected onto a standard brain schematic (Movie 1). We used the Connectome Workbench software to generate these visualizations (Marcus et al., 2011).

We used the CAB-NP (Ji et al., 2019), plus adjustments derived from the empirical resting-state fMRI data of the participants studied herein, as the predefined reference (Fig. 3). This a priori network partition can be thought of as a minimal parameter space maintained by deviation in the present study; however, future work may apply community detection (i.e., empirically-based network partition) if appropriate. Deviation may be accompanied by its complementary measure, network partition adherence, which was the relative frequency of states in which a given region adhered to its predefined network assignment (or 1-deviation, meaning that deviation and adherence add up to 1 , or $100 \%$ of task states). We further unpacked deviation by depicting which networks were preferred by regions (when deviating from the partition), generating reassignment profiles.

\section{Cognitive control cartographies}

We rendered one primary mapping of cross-state network-reconfiguration properties and two secondary mappings which broke up the primary mapping's properties into within-network and between-network scores. For the secondary mappings, within-network GVC was computed by setting between-network FC estimates to "NaN" (i.e., "not a number" in MATLAB) before inputting data into the GVC algorithm (Fig. 5A). Likewise, between-network GVC was computed by setting within-network FC estimates to $\mathrm{NaN}$ (Fig. $5 B$ ). This effectively nullified the variability for those regions such that GVC ignored them during computation (which principally employs SD across states; Fig. 4A; Table 3). Between-network GVC was equivalent to BVC described above. Within-network deviation was computed by setting FC estimates of

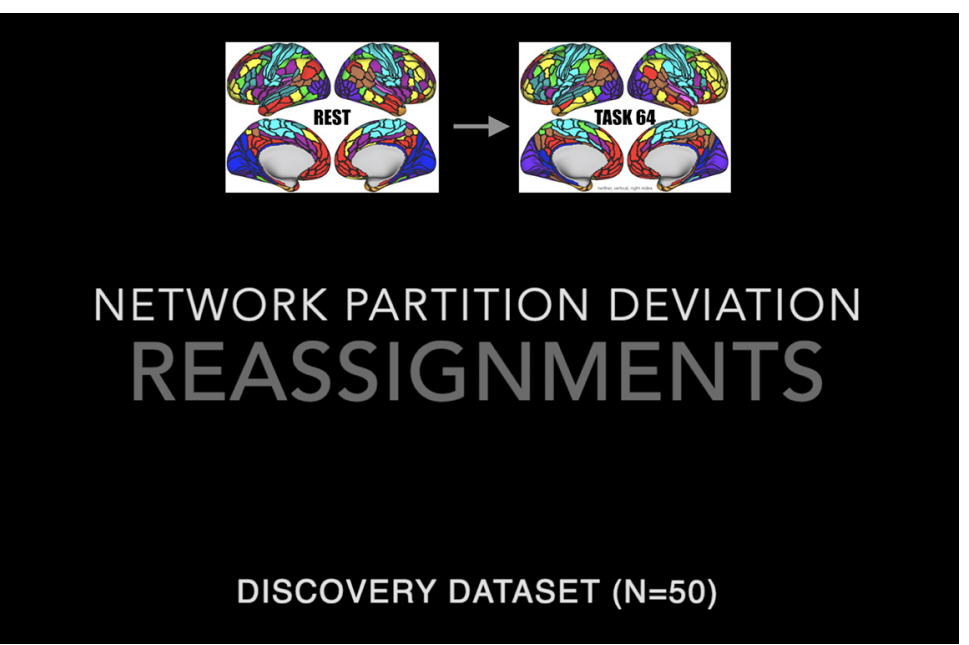

Movie 1. Video depiction of cortical network reassignments across task states, computed via network partition deviation. The video depicts each region's network "preference" observed during the computation of network partition deviation, across all 64 C-PRO task states, and for $n=50$ discovery dataset participants (see Materials and Methods). Briefly, per task state and region, the maximum connectivity estimate was found, (Fig. 4B; Table 3). These network indices were then mapped back onto brain schematics to visualize how devition defines network reassignment across tasks. This video also depicts the dynamics captured by deviation. ondary visual networks, shown mainly in occipital regions as blue and purple, respectively). However, some NP naming system (Ji et al., 2019; Fig. 3). [View online]

between-network regions to the rsFC for those regions before inputting data into the deviation algorithm (Fig. $5 \mathrm{C}$ ). Likewise, for between-network deviation, we substituted within-network estimates with corresponding regions' rsFC (Fig. 5D). We considered the use of rsFC most appropriate given deviation's inherent comparison to the resting-state partition. Thus, deviation away from the resting-state partition would always be zero for regions set to their resting-state estimates. Figure 5 visually depicts the input data schemes for each of these secondary cartographies.

\section{Decoding analyses}

A classification analysis was performed for each functional network to test whether network connectivity patterns could be used to significantly decode task state. As in Cole et al. (2013b), three four-way classifications 


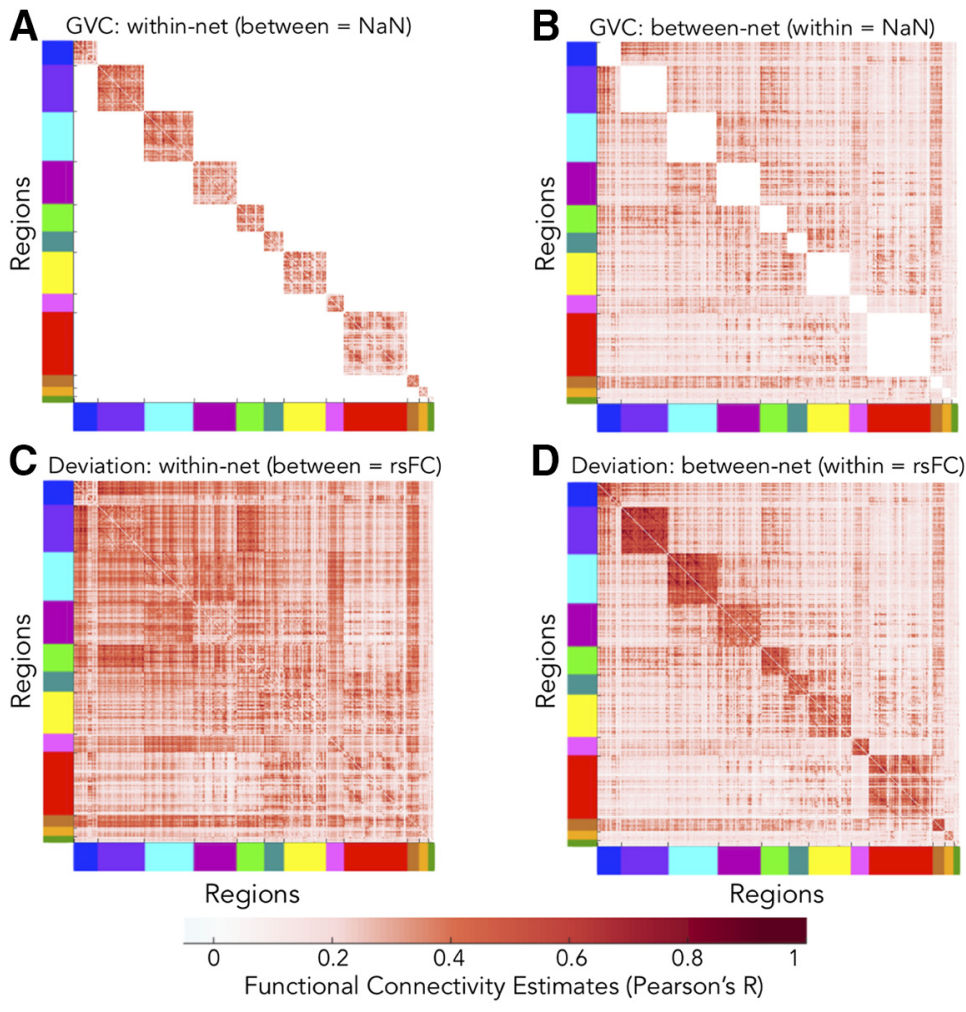

Figure 5. Input data schemes used in computing measures of the secondary cognitive control cartographies. Data refers to FC estimates (Pearson's $R$; colored according to the bottom-most scale). Put another way, each panel contains modified correlation matrices. The cross-state means are visually represented, but analyses included all $64 \mathrm{C}$ PRO task states. Axes are color coded according to the empirically-adjusted CAB-NP (Fig. 3). A, Representation of input data for within-network GVC. Between-network FC estimates were set to NaN (white). B, Input data for between-network GVC. Within-network FC estimates were set to NaN (white). C, Input data for within-network deviation. Between-network FC estimates were set to their resting-state values. $\boldsymbol{D}$, Input data for between-network deviation. Within-network FC estimates were set to their resting-state values.

were performed using connectivity patterns from each network separately. Unlike Cole et al. (2013b), we extended the cohort to include $n=50$ (per discovery and replication datasets), used correlation as a classifying distance measure (Haxby et al., 2001; Mur et al., 2009; Ito et al., 2017) and performed 8-fold cross validation. We performed within-subjects classifications. Each subject had 64 samples of task-state connectivity estimates for each distinct task rule set (Fig. 1; see above, FC estimation). Of those 64 samples, classifiers were trained on a random subset (over 8-fold) of 56 task states and tested on the remaining (held out) eight task states. Each task state was a combination of three rule domains: logic, sensory, and motor. For each of the three decoding analyses, we isolated specific rules from each of these domains. Therefore, the labels associated with these states were according to: (1) logic (both, not both, either, or neither), (2) motor (left middle, left index, right middle, or right index), and (3) sensory (vertical, red color, high pitch, constant tone) rule-set domains (Fig. 1). Therefore, chance accuracy was $25 \%$ in each four-way analysis. We averaged task-state connectivity patterns (i.e., features) across identical training-set labels (e.g., in the logic rule-set classification: training-set connectivity estimates that contained "both" were averaged). We used a minimum-distance classifier (based on Spearman's rank correlation score), where a test set would be classified as the rule type whose centroid was closest in multivariate space (Mur et al., 2009). We compared these distances for each set of matched versus mismatched training and test set labels. When a matched similarity score was larger than all mismatched similarities, this was deemed an accurate decoding. To summarize, decoding accuracy was the percent of rules accurately decoded (in each of the three rule set domains), averaged across 8-folds (Varoquaux et al., 2017).

In order to assess cross-subject statistical significance of the decoding accuracies of each network, we performed right-tailed Student's $t$ tests against chance accuracy. We then used the Max-T nonparametric permutation testing approach (1000 permutations) to address multiple comparisons (for details, see above, Experimental design and statistical analysis). In each permutation, rule-set labels were randomly shuffled before the classification analysis was performed. A null distribution of decoding accuracies and corresponding $t$ statistics was built and used to assess statistical significance.

\section{Code and software accessibility}

We included all MATLAB, python, and demo code in a publicly available platform. Data are available at the level of FC estimation, for the use of loading into demo scripts. Data at other levels of processing, or data otherwise presented in this study, are available on request. The master GitHub repository for this study can be found here: https://github.com/ColeLab/ controlCartography.

\section{Results}

Intrinsic and task-state FC

Replicating previous findings (Cole et al., 2014), cortex-wide rsFC (Fig. 6A) and tFC (Fig. 6B) estimates were highly similar. This significant similarity was observed for the average tFC taken across all 64 C-PRO tasks (Mantel- $r=0.89$, $p<0.0001, R^{2}=0.79$ ), as well as for each C-PRO rule individually (Table 4 ). This aligns with previous observations that the set of networks present during rest are highly related to the set of networks present during task states. In addition to the minimal cognitive demands during rest providing a cognitive baseline for a variety of tasks, this result suggests that rest may be an appropriate intrinsic reference state for characterizing changes in networks across multiple task states. The similarity observed between rsFC and tFC (all 64 C-PRO tasks) in the replication dataset was comparable (Mantel- $r=0.90$, $p<0.0001, R^{2}=0.81$ )

To summarize changing connectivity from the resting state to the average task state, we created a task versus rest difference matrix, and found $21 \%$ of those values to be significant differences ( $\max -\mathrm{T}$ critical threshold $=5.46,5000$ permutations). The finding that rsFC and tFC (across multiple task states) are highly correlated, yet the differences between them are nontrivial, justified subsequent analyses of functional reconfigurations between these two kinds of states. Findings were consistent in the replication dataset: $\sim 32 \%$ significant rest-to-task differences (max-T critical $=5.67$ ).

\section{Network metrics: variability coefficients}

Our prior work found that the FPN contains flexible hub regions, network nodes capable of rapid reconfiguration with changing task demands (flexible) that have extensive connectivity (hubs; Cole et al., 2013b). Recent work has suggested that the CON also contains hub-like regions (Power et al., 2013; Ito et al., 2017), yet it is unknown if they are likewise flexible. Accordingly, we used two related metrics to assess whether networks contain flexible hub regions (Cole et al., 2013b): GVC, and BVC (Fig. 4A; Table 3).

Critically, Cole et al. (2013b) only involved $N=15$ subjects, compared with the $n=50$ discovery and separate $n=50$ replication datasets in the present study. Thus, replicating the results of 
Cole et al. (2013b) would be nontrivial. Replicating the main result of Cole et al. (2013b), regions of the FPN had the highest GVC (Fig. 7A) and BVC compared with the mean of all other networks (GVC: $\max -\mathrm{T}_{(49)}=10.94, p<0.0001$; BVC: $\max -$ $\left.\mathrm{T}_{(49)}=10.69, p<0.0001\right)$. BVC and GVC results were highly correlated at both the network (Fig. 7A) and regional levels (Fig. 7B; network-wise: $r=0.9912$, $p<0.00001, R^{2}=0.9824$ cross-network shared variance; region-wise: $r=0.9972, p<0.0001, R^{2}=0.9944$ cross-region shared variance), suggesting that withinnetwork estimates do not dominate the outcome of GVC analyses.

These results were replicated in the replication dataset: the FPN demonstrated the highest GVC (Fig. $7 F)$ and BVC compared with the mean of all other networks (GVC: $\max -\mathrm{T}_{(49)}=7.23, p<0.0001$; BVC: $\left.\max -\mathrm{T}_{(49)}=6.93, p<0.0001\right)$. BVC and GVC results were also tightly correlated at both the network and regional levels in the replication dataset (networkwise: $r=0.9925, p<0.00001, R^{2}=0.985$ cross-network shared variance; region-wise: $r=0.9975, p<$ .0001, $R^{2}=0.995$ cross-region shared variance). Additionally, both GVC and BVC results highly overlapped between discovery and replication datasets (GVC: Rho $=0.9091, p<0.00001$; BVC: Rho $=0.881$, $p=0.0002)$.

In conjunction with many studies reporting increased FPN activity as a function of cognitive control demands (Yeo et al., 2015), this pattern of results supports the notion that the FPN contains flexible regions adaptively configured for multitask control. Further, we compared GVC between control networks and each of the other networks. FPN regions were significantly higher than each other network (using the max-T approach, $p<0.0001$ ), except for the ventral multimodal network. CON regions were significantly different (typically lower) than each other network on the measure of GVC (using the max-T approach, $p<0.0001$ ), except for posterior multimodal and ventral multimodal networks. Lastly, a paired-samples $t$ test comparing FPN and CON revealed a significant difference in GVC scores $\left(t_{(49)}=11.68, p<0.00001\right)$, suggesting that the two proposed control networks exhibit distinct variability of global connectivity. In the replication dataset, FPN regions' GVC scores were also significantly higher than each other network, except for the ventral multimodal network (Fig. $7 F$, orange bar). CON regions were significantly different from each other network on the measure of GVC in the replication dataset, with no exceptions (Fig. 7F). The paired-samples $t$ test contrasting FPN and CON specifically also showed a significant difference on GVC scores in the replication dataset $\left(t_{(49)}=10.55\right.$, $p<0.00001$ ).

Despite evidence that FPN has strong global variability consistent with flexible hubs, it remains unclear whether that variability is systematically related to task information content, a prerequisite for flexible hubs to implement task-related reconfigurations. Prior findings (Cole et al., 2013b) demonstrated that FPN connections systematically vary with increasing task-state similarity. We sought to replicate this result in FPN and, given the current focus on cognitive control systems, we additionally analyzed CON connections. As in Cole et al. (2013b), task-state similarity was taken as the number of overlapping, or shared, rules presented to participants, across all 64 tasks (Fig. 7C; also
Table 4. Summary of the similarities between intrinsic rsFC and multitask FC

\begin{tabular}{llll}
\hline $\begin{array}{l}\text { Task FC data: } \\
\begin{array}{l}\text { C-PRO rule } \\
\text { set }\end{array}\end{array}$ & $\begin{array}{l}\text { Correlation } \\
\text { with rest } \\
\text { (Mantel-r) }\end{array}$ & $\begin{array}{l}p \text { value } \\
\text { (permutation } \\
\text { testing) }\end{array}$ & $\begin{array}{l}\text { Shared } \\
\text { variance } \\
\left(R^{2}\right)\end{array}$ \\
\hline $\begin{array}{l}\text { Sensory rules } \\
\quad \text { Vertical orientation }\end{array}$ & 0.7966 & $3.05 \times 10^{-8}$ & 0.6345 \\
$\quad$ Red color & 0.7918 & $3.17 \times 10^{-8}$ & 0.6269 \\
$\quad$ High pitch sound & 0.7964 & $3.10 \times 10^{-8}$ & 0.6343 \\
$\quad$ Constant tone & 0.7864 & $3.24 \times 10^{-8}$ & 0.6185 \\
Motor rules & & & \\
$\quad$ Left index finger & 0.7847 & $3.26 \times 10^{-8}$ & 0.6157 \\
$\quad$ Right index finger & 0.7966 & $3.60 \times 10^{-8}$ & 0.6345 \\
$\quad$ Left middle finger & 0.7939 & $3.14 \times 10^{-8}$ & 0.6302 \\
$\quad$ Right middle finger & 0.7844 & $3.33 \times 10^{-8}$ & 0.6153 \\
Logic rules & & & \\
$\quad$ Both & 0.7983 & $3.04 \times 10^{-8}$ & 0.6372 \\
$\quad$ Either & 0.7934 & $3.74 \times 10^{-8}$ & 0.6295 \\
$\quad$ Not both & 0.7833 & $3.28 \times 10^{-8}$ & 0.6136 \\
$\quad$ Neither & 0.7966 & $4.62 \times 10^{-8}$ & 0.6345 \\
\hline
\end{tabular}

Each row lists a task-state (i.e., $\mathrm{FFC}$ ) comparison to rsFC (Fig. 6A). These tFC estimates were based on each of the 12 C-PRO rules (see Fig. 1) and averaged across participants. Columns list the Mantel-r statistic, corresponding $p$ value in scientific notation (nonparametric permutation testing; see Materials and Methods and Glerean et al., 2016) and shared variance $\left(R^{2}\right)$. Connectivity of all C-PRO rule sets significantly correlated with rsFC.

see Materials and Methods). We then measured Spearman's rank correlations (as a score of similarity) among connections according to these task-state pairings, for both FPN (Fig. 7D) and CON regions (Fig. $7 E$ ). An approximately linear relationship was observed, suggesting that shifts in connectivity systematically 


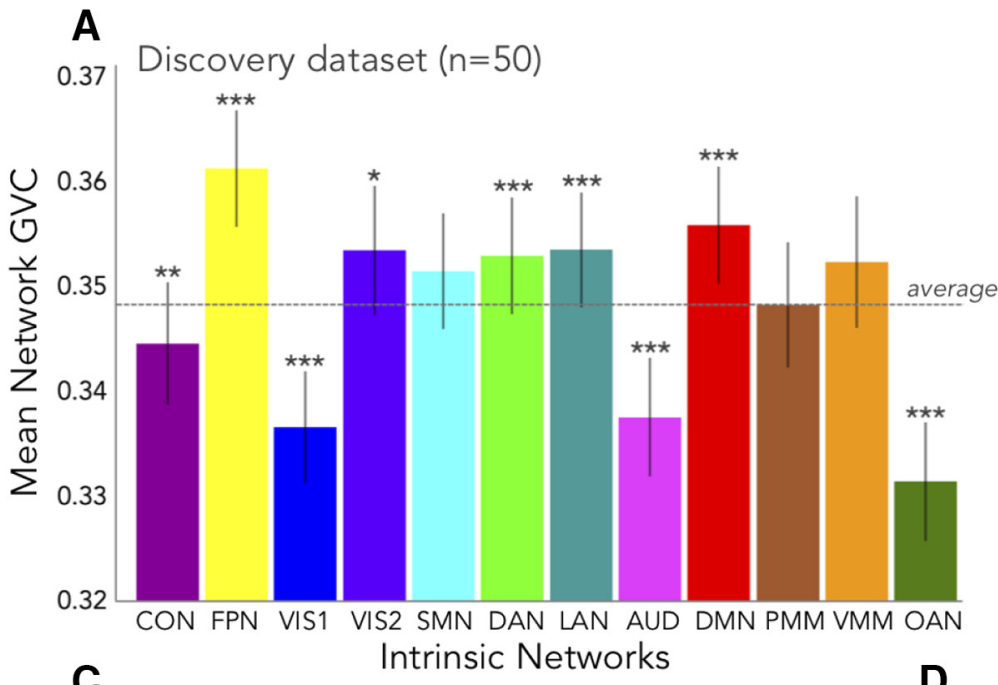

C

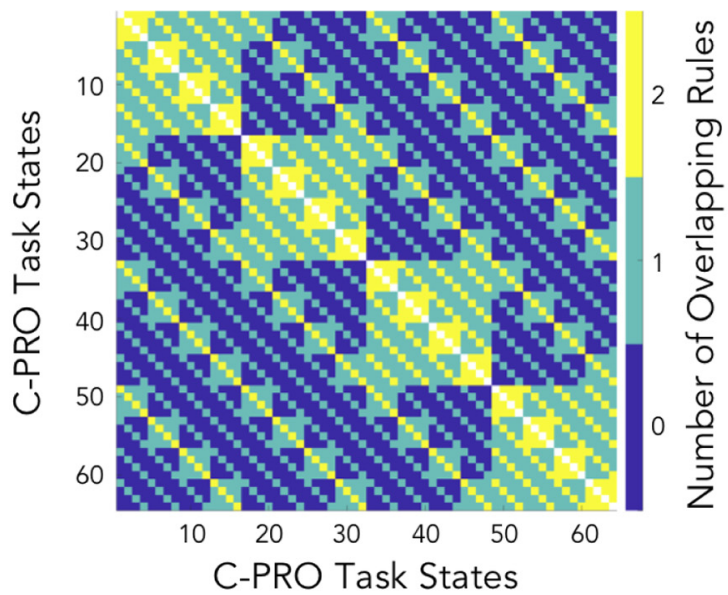

F

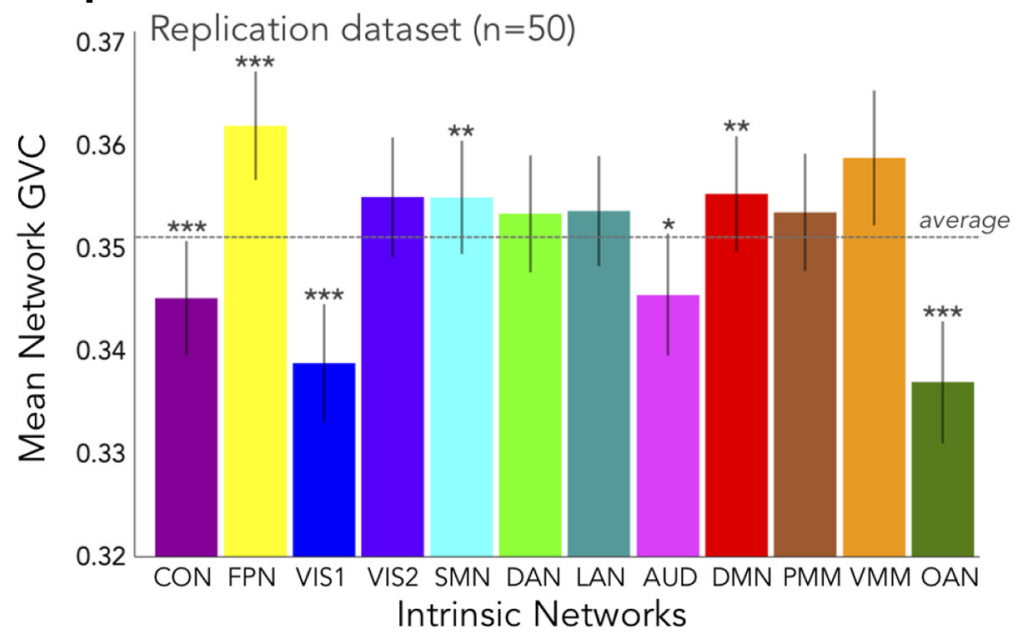

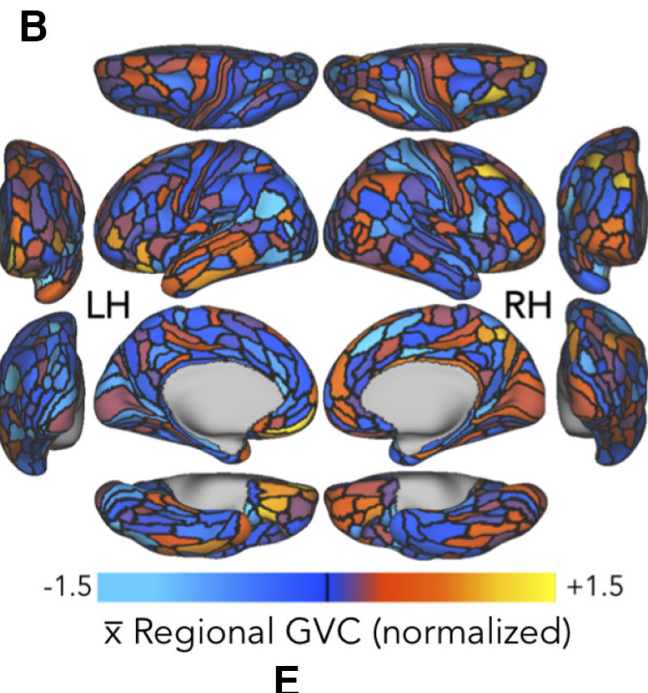

0.1278

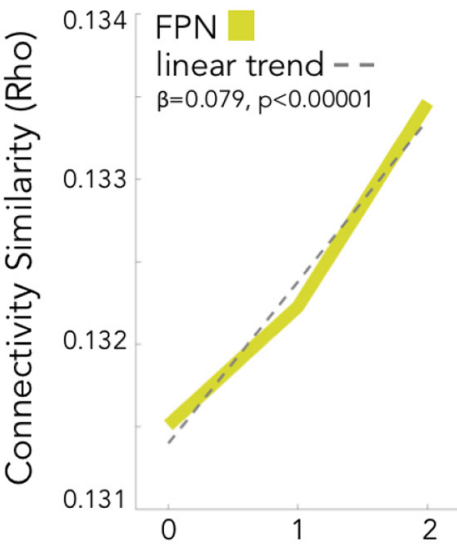

linear trend - $\beta=0.076, p<0.00001$

0.127

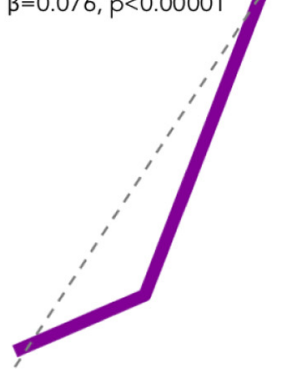

0.1254

0

Task Similarity (number of overlapping rules)

G

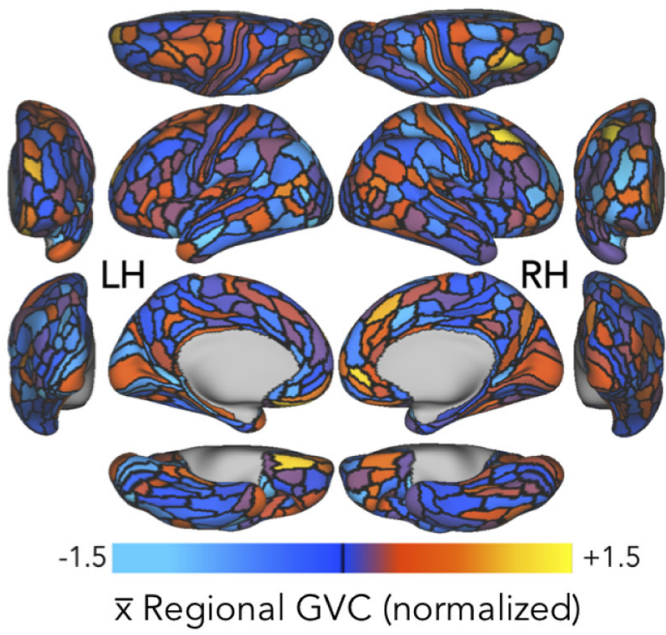

Figure 7. GVC. A, Network-mean GVC, across discovery dataset participants and all C-PRO task states. Error bars: SEM. Asterisks: statistically significant $t$ tests, using the max-T approach (see Materials and Methods). ${ }^{*} p<0.05 ;{ }^{* *} p<0.005 ;{ }^{* * *} p<0.0005$. Horizontal, dashed line: average GVC across networks. $\boldsymbol{B}$, Regional-mean GVC, projected onto a cortical surface. LH $=$ left hemisphere, RH = right hemisphere. C, Similarity of C-PRO task states, represented by number of overlapping rules ( 0 overlapping rule $=$ blue, 1 overlapping rule $=$ green, 2 overlapping rules $=$ yellow). An overlap of three rules exists along the diagonal (white), but these connections were not included in analyses because connectivity similarity would be Rho $=1$ (identical task states). $\boldsymbol{D}$, Relationship between FPN connectivity similarity and task similarity. All connectivity estimates were included. Gray dashed line: linear trend, with associated $\beta$ and $t$ test significance listed. $\boldsymbol{E}$, Same as $\boldsymbol{D}$ but for CON regions. The results in $\boldsymbol{D}, \boldsymbol{E}$ demonstrated that control network connectivity similarity varied systematically with task similarity, suggesting that GVC results $(\boldsymbol{A}$, $\boldsymbol{B}$ ) were not driven by network noise. $\boldsymbol{F}$, Same as $\boldsymbol{A}$, but replication dataset GVC results at the network level. $\boldsymbol{G}$, Same as $\boldsymbol{B}$, but replication dataset GVC results at the regional level. GVC results highly overlapped between discovery $(\boldsymbol{A})$ and replication $(\boldsymbol{F})$ datasets: $R h 0=0.9091, p<0.00001$. 

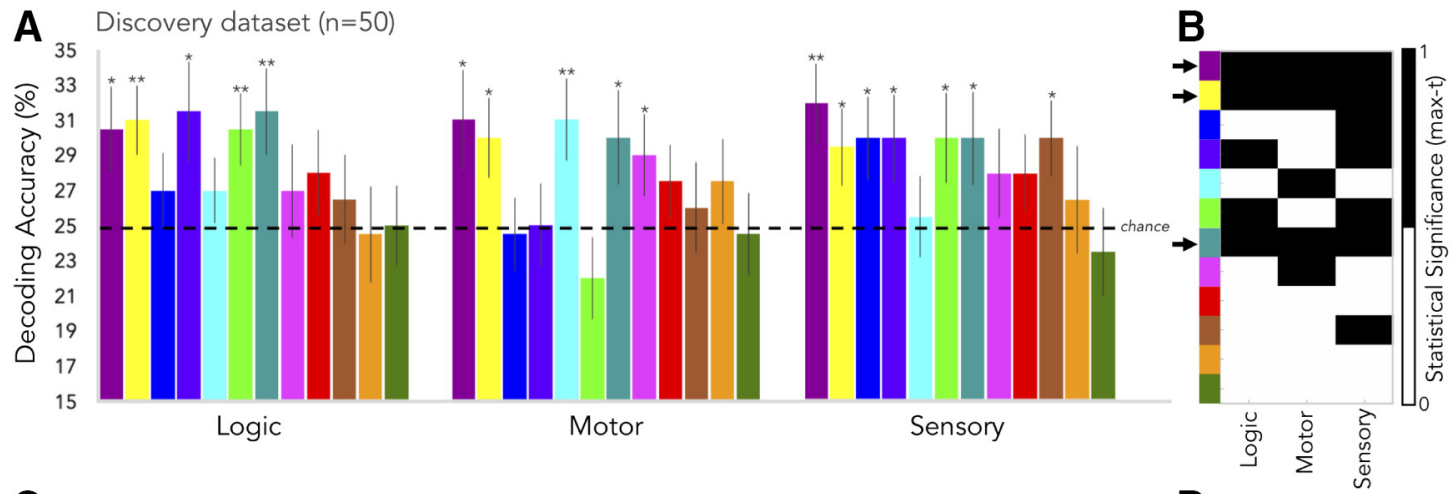

$\mathbf{E}$

CAB-NP Network Colors

Cingulo-Opercular (CON) Frontoparietal (FPN) Primary Visual (VIS1) Secondary Visual (VIS2) Somatomotor (SMN)

Dorsal Attention (DAN)
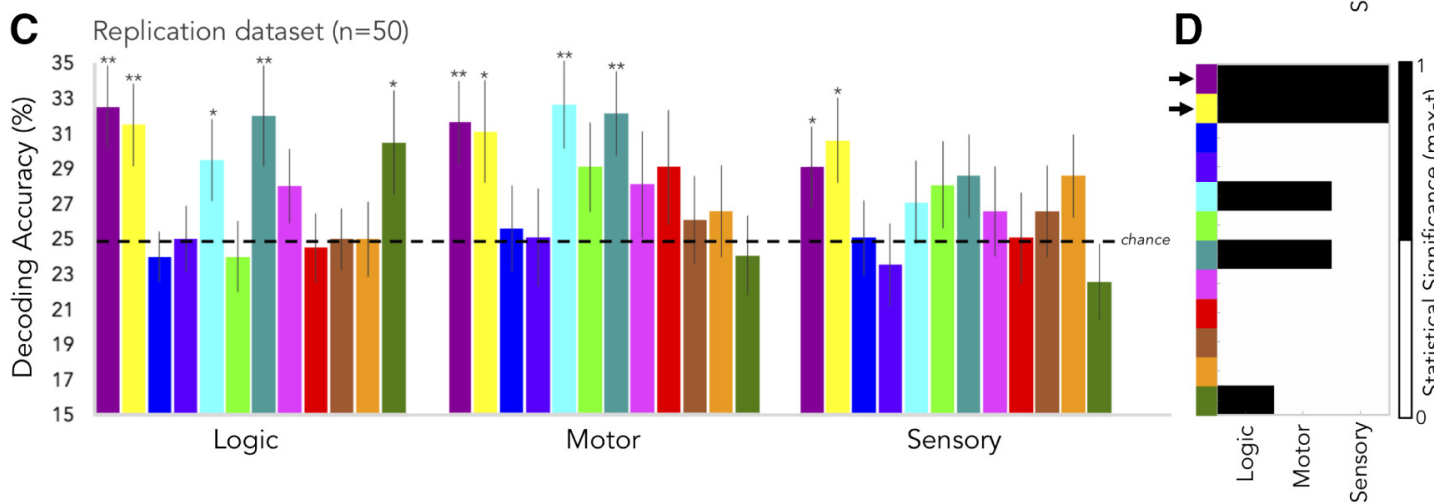

Language (LAN) Auditory (AUD) Default (DMN) Posterior Multimodal (PMM)

Ventral Multimodal (VMM)

Orbito-Affective (OAN)

Figure 8. Decoding task rule information with task-state connectivity. $\boldsymbol{A}$, Cross-subject (discovery dataset) network-mean decoding accuracies for logic, motor, and sensory rules (four-way classifications where chance accuracy was $25 \%$, represented by the horizontal, dashed line). Error bars: SEM. Asterisks: statistically significant $t$ tests using the max-T approach (see Materials and Methods). ${ }^{*} p<0.05 ;{ }^{* *} p<0.005$. B , Statistical significance tallies (binarized: black = significant or 1 , white $=$ not significant or 0 ) for each network ( $y$-axis, color-labeled) and each rule set ( $x$-axis) for the discovery dataset. Black arrows to the left of $y$-axis color labels mark networks that significantly decoded all three types of rules, which included the control networks, CON and FPN, as well as the language network (LAN). C, Same as in $\boldsymbol{A}$ but for the replication dataset. $\boldsymbol{D}$, Same as in $\boldsymbol{B}$ but for the replication dataset. The control networks, CON and FPN, were the only networks to significantly decode all three rule types in the replication dataset. LAN came close (as in $\boldsymbol{B}$ ), but its statistical significance did not survive the permutation testing procedure for sensory rules. $\boldsymbol{E}$, The CAB-NP color scheme used across all panels $\boldsymbol{A}-\boldsymbol{D}$ (as in Fig. 3 but rearranged to highlight the cognitive control networks). Both FPN and CON connectivity patterns significantly decoded task rules above chance, demonstrating their importance in task representation.

relate to shifts in task state, and are not simply a by-product of noise. Note that at the subject level, the effect size of shifting connectivity is not interpretable because it is unknown how many connection changes are required to cognitively implement a task-rule change (e.g., two robust connection changes may be enough cognitively, but produce small correlation changes at the network level). The linear regression weights of these similarity scores were consistently different from zero across subjects (FPN: $t_{(49)}=35.51, p<0.00001$; CON: $\left.t_{(49)}=33.25, p<0.00001\right)$. Next, we performed the same analyses, but restricted the connections to those maintaining the highest variability (across top 2$10 \%$ in steps of $2 \%$ ) across task states (i.e., the "VC" of GVC) for both the FPN (as in Cole et al., 2013b) and CON. Results were similar to the main results across all thresholds, with linear weights significantly different from zero $(p<0.05)$. These results suggest that GVC results are likewise driven by systematic changes in connectivity, and not network noise. These results additionally reveal that $\mathrm{CON}$ also systematically changes its global connectivity pattern according to task goals, although the GVC results suggest these systematic changes are smaller in CON (and most other networks) than FPN.

Next, we tested the hypothesis that global FPN and CON connectivity patterns were specific enough to each task set that they could be used to reliably predict the current task rules being used. As in Cole et al. (2013b), FPN (as well as all other networks in the present study) features were restricted by their somatomotor network (SMN) connections in the tests of motor-domain rule classification. We tested how well control network connectivity patterns could be used to decode rule sets in the three C-PRO domains (logic, sensory, and motor; Fig. 1) by assessing task decodability of every CAB-NP network (Fig. 3) with nonparametric permutation testing to address multiple comparisons (see Materials and Methods). In each domain, there were four distinct rules, thus chance accuracy was 25\%.

Consistent with our hypothesis that FPN and CON are especially important for network-level representation of task information, FPN and CON were the only two networks whose connectivity patterns could be used to decode all three rule domains across both discovery and replication datasets (Fig. 8; $p<0.05$, nonparametrically corrected for multiple comparisons). The connectivity patterns of other networks could be used to decode task information in a more limited manner, often for functionally-relevant task domains (e.g., SMN for motor rules; Fig. $8 A, C)$. To clarify the pattern of task-rule decoding results across control and non-control networks, we generated binarized matrices depicting statistical significance (Fig. 8B,D). This allowed us to more easily observe which networks' cortical connectivity patterns could be used to significantly decode rule sets across all three C-PRO task domains (Fig. $8 B, D$, arrows). In the discovery dataset, the cognitive control networks, FPN and CON, plus LAN were the only networks that decode all rule sets. In the replication dataset, only the FPN and CON could significantly decode all rule sets. It is worth noting that LAN came close to maintaining decodability across all rule types in the replication dataset as well but did not survive correction for multiple comparisons in the 


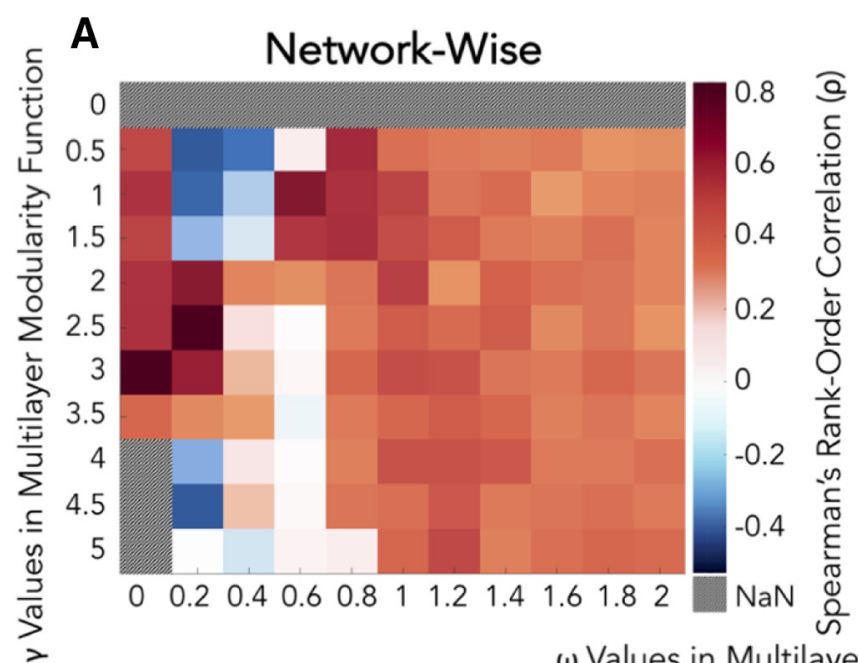

\section{B Region-Wise}

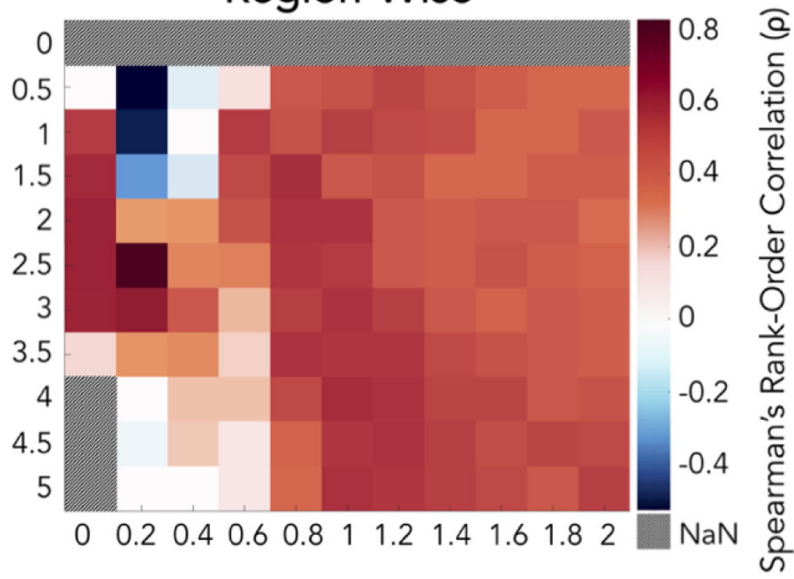

$\omega$ Values in Multilayer Modularity Function

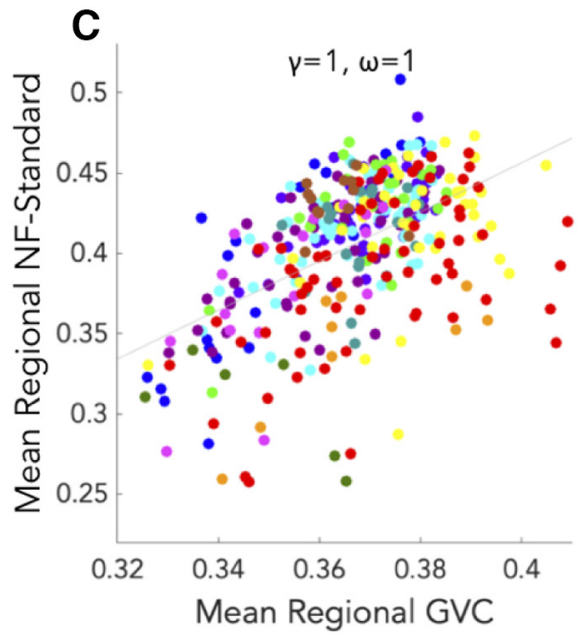

D

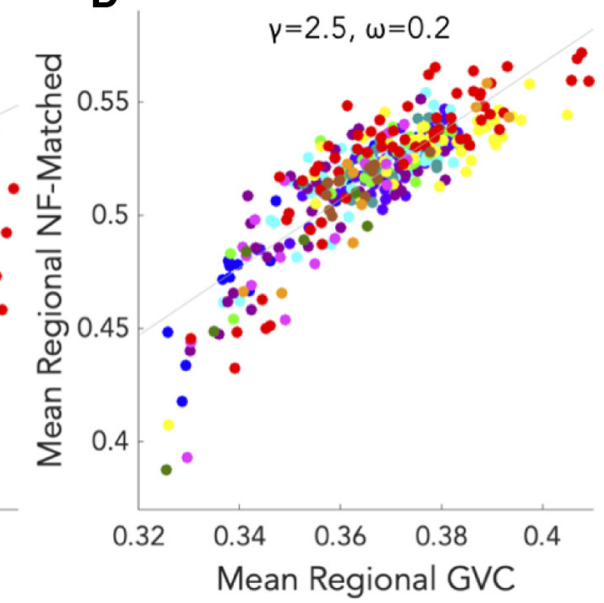

$\mathbf{E}$

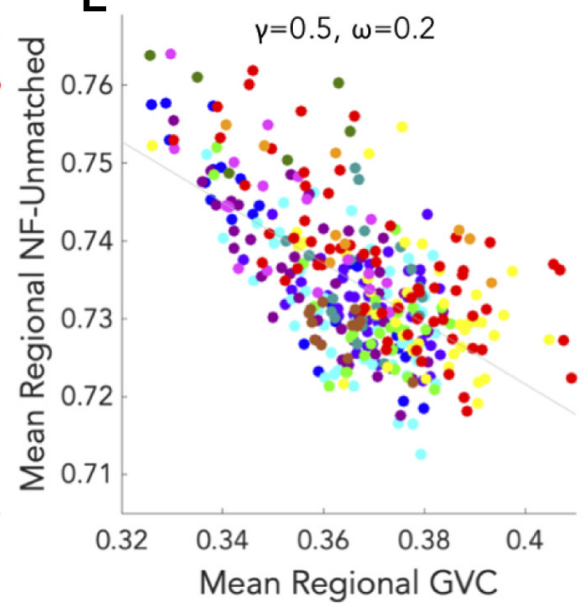

Figure 9. Comparison of GVC and NF, discovery dataset. $A$, Network-wise comparisons (Spearman's rank order correlation) of GVC and NF swept by multilayer modularity parameters, across participants. $\boldsymbol{B}$, Same as $\boldsymbol{A}$ but at the region-wise level. $\boldsymbol{C}$, Regional mean GVC plotted over regional mean NF-standard (multilayer modularity parameters: $\gamma=1$ and $\omega=1$ ). NF-standard results were yielded by the standard parameter combination. Each region (mean across $n=50$ participants) was plotted as individual scatter points and color-coded according to the network it belongs to (as in Fig. 3). $\boldsymbol{D}$, Same as ( but with NF-matched represented on the $y$-axis ( $\gamma=2.5$ and $\omega=0.2)$. This parameter combination yielded NF results most correlated with GVC (or "matched"), as depicted in $A, B$ by dark red squares. $\boldsymbol{E}$, Same as $\boldsymbol{C}, \boldsymbol{D}$ but with NF-unmatched represented on the $y$-axis $(\gamma=0.5$ and $\omega=0.2)$. This parameter combination yielded NF results least correlated with GVC (or "unmatched"), as depicted in $\boldsymbol{A}, \boldsymbol{B}$ by blue squares. The variable results in panels $\boldsymbol{C}-\boldsymbol{E}$ motivated the need for a new measure of network reconfiguration that was less linked to parameter selection.

sensory domain $\left(t_{(49)}=1.48, p=0.08\right)$. Decodability of task information in the language network is consistent with all of the C-PRO rules having been cued with words (Fig. 1). The tendency for control networks' global connectivity patterns to so consistently carry task rule information in all three domains suggests that their network interactions likely carry information critical to task representation.

\section{Network metric: flexibility}

NF measures functional network dynamics related to task-state time series (Mucha et al., 2010; Bassett et al., 2011, 2013a,b; Cole et al., 2014) and is thus highly relevant to our current hypotheses regarding control network reconfigurations. Conceptually, NF is aligned with GVC, particularly as both quantify large-scale changes in functional network configurations. However, it is unknown whether these metrics capture the same aspects of network reconfiguration. The computations of both GVC and NF are oriented around a measure of network change, however, the approaches are distinct enough to hypothesize that NF and GVC will not entirely overlap. We hypothesized that differentiation between NF and GVC would lend insight into the nature of control network reconfiguration. In particular, GVC assesses continuous changes in connectivity strengths, while NF assesses discrete network reassignments.

The multilayer modularity step required parameters $\omega$ (temporal resolution or "coupling") and $\gamma$ (spatial resolution) to be chosen. The standard values used for these parameters across multiple studies are $\gamma=1$ and $\omega=1$ (Bassett et al., 2013b; Braun et al., 2015; Chen et al., 2015). NF that resulted from community detection at $\gamma=1$ and $\omega=1$ was termed NF-standard. Since there is only a limited theoretical basis for those parameter choices, we computed NF across a range of values around these standards, such that $\gamma$ was varied between zero and five in steps of 0.5 ; and $\omega$ was varied between zero and two in steps of 0.2 (Fig. 9A,B). These sweeps resulted in 121 vectors of regional NF estimates (per participant). It was clear that results depended substantially on the exact values of $\gamma$ and $\omega$, such that we were unable to make systematic inferences regarding flexibility of network assignments using NF. To illustrate this: we identified parameters $(\gamma=2.5$ and $\omega=0.2)$ that yielded high cross-node 
similarity to GVC, termed NF-matched [discovery dataset: $R h o=0.8169, p<0.00001$ (Fig. 9D); replication dataset: Rho= $0.6993, p=0.015$ ] and others $\gamma=0.5$ and $\omega=0.2$ that yielded a negative relationship with GVC, termed NF-unmatched [discovery dataset: $R h o=-0.4546, p=0.14$ (Fig. 9E); replication dataset: $R h o=-0.3077, p=0.34$, while the NF-standard parameters ( $\gamma=1$ and $\omega=1$ ) yielded a positive but not significant relationship with GVC [discovery dataset: $R h o=0.4825, p=0.12$ (Fig. 9C); replication dataset: $R h o=0.3147, p=0.32$ ].

\section{Network metric: partition deviation}

Given that NF demonstrated inconsistent results dependent on tuning parameters, we created a novel metric, network partition deviation (or simply, deviation), that could quantify network reconfiguration without the need for a parameter search. Deviation enumerates network reassignments across task states by quantifying the percent of states (i.e., the relative frequency across tasks) in which a given region deviates from a predefined partition (see Materials and Methods; Fig. 4B; Table 3; Movie 1). We employed the CAB-NP adjusted by the empirical resting-state data of participants herein (Fig. 3) as the intrinsic, predefined reference. See prior work (Cole et al., 2014; Krienen et al., 2014) and above, Intrinsic and task-state FC, for evidence that resting state provides an appropriate intrinsic network configuration to act as a reference for assessing network deviations.

Of the cognitive control networks of interest here, the CON and the FPN, the CON displayed the highest mean deviation, which was significantly higher than the mean across all other networks $\left(\max -\mathrm{T}_{(49)}=12.74, p<0.0001\right.$; Fig. $\left.10 \mathrm{~A}\right)$. Moreover, the FPN demonstrated deviation that was near the mean, and was not significantly different from the mean across all other networks. This contrasted from the conclusions drawn from GVC, which showed the FPN significantly above the mean, and the CON significantly below it. Similarly in the replication dataset, CON regions' deviation scores were again significantly higher than the mean of all other networks $\left(\max -\mathrm{T}_{(49)}=16.33\right.$, $p<0.0001$; Fig. 10E).

Next, we performed planned contrasts of the control networks' deviation scores versus each other networks' deviation scores, using the max-T method to correct for multiple comparisons (see Materials and Methods). The CON's deviation was significantly higher than every other network $(p<0.0001)$, except for the orbito-affective network. Deviation estimates of FPN regions were significantly different from about half of the other networks $(p<0.0001)$, including: secondary visual, somatomotor, cinguloopercular, auditory, posterior multimodal, ventral multimodal, and orbito-affective. The deviation of FPN and CON regions were significantly different $\left(t_{(49)}=6.28, p<0.00001\right)$, suggesting that the control networks differ on how often they deviate from their intrinsic partitions across task states. In the replication dataset, CON regions' deviations scores were significantly higher than each other network, except for the orbito-affective network. Lastly, the paired samples $t$ test to compare the deviation of CON and FPN in the replication dataset likewise showed a significant difference $\left(t_{(49)}=9.13, p<0.00001\right)$.

To further explore the task-state reconfiguration property that deviation was capturing, we generated "reassignment profiles" at both the network (Fig. 10C) and region (Fig. 10D) levels. Reassignment profiles showed precisely which networks were preferred when a region was deviating from the intrinsic partition. As shown in Figure $10 C$, the CON deviated to many other networks in an evenly-distributed manner (relative to other networks' reconfigurations) with some bias to somatomotor connections, whereas the FPN deviated less overall and with more specific preferences, favoring the dorsal attention, language, and default networks (in addition to itself). As with other graph metric results, deviation estimates highly overlapped between discovery and replication datasets (Rho $=0.958$, $p<0.00001)$, as did reassignment profiles (Rho $=0.848$, $p<0.0001)$.

\section{Network cartographies}

We found that FPN regions expressed high GVC yet relatively low deviation. In contrast, CON regions displayed lower GVC yet higher deviation. Altogether, the CON and the FPN both exhibited higher reconfiguration properties than other networks. However, the diversity of findings across network metrics suggested that composite, multidimensional profiles were warranted to fully map out their functionalities. Careful examination of network metric estimates for $\mathrm{CON}$ and FPN regions clarified the pattern of results. FPN connectivity tended not to deviate (and when it did, to only a small number of networks, as in Fig. 10C, $D$ ), whereas CON connectivity was more uniform (or "evenly" deviating, as in Fig. 10C,D). Figure $11 \mathrm{~A}$ depicts prominent network properties in a cartographic manner (Guimerà and Nunes Amaral, 2005; Mattar et al., 2015), charting GVC on the $y$-axis against deviation on the $x$-axis. The FPN can be found in the upper right quadrant of this cartography, near the mean demarcation line for deviation (Fig. $11 \mathrm{~A}$, vertical gray line), pointing to the high-variability yet low-deviation performance of FPN regions in response to cognitive control task state changes. The CON can be found in the lower right quadrant of the cartography in Figure $11 A$, suggesting a low-variability yet high-deviation complement to FPN in properties supporting cognitive control.

To expand on the primary mapping in Figure $11 \mathrm{~A}$, we generated two secondary cartographies that depict the quantification of each primary measure's within-network and between-network scores (see Materials and Methods). Briefly, within-network dynamics were assessed by keeping the between-network connectivity fixed across states (defined by resting state). Similarly, between-network dynamics were assessed by keeping the withinnetwork connectivity fixed across states (defined by resting state; for data input schematics, see Fig. 5). Figure $11 B$ charts these dimensions of GVC, showing both within-network and between-network FPN connections to be high on global variability and CON to be near-mean on both within-network GVC and between-network GVC. Figure $11 C$ depicts within-network and between-network deviation. Both FPN and CON regions were near the mean for between-network deviation (yet on opposing sides of the mean; Fig. 11C, vertical gray line), yet low and high, respectively, for within-network deviation. This suggests that CON's high deviation was driven primarily by changes in within-network connectivity.

Supporting dissociation of FPN and CON in terms of network dynamics, we directly compared FPN and CON regions on each secondary cartographic metric (Fig. 11B,C), and found the following: (1) FPN was significantly higher than CON on withinnetwork GVC $\left(t_{(49)}=8.92, p<0.00001\right)$; (2) FPN was significantly higher than CON on between-network GVC $\left(t_{(49)}=11.43\right.$, $p<0.00001$ ); (3) CON was significantly higher than FPN on within-network deviation $\left(t_{(49)}=6.55, p<0.00001\right)$; and (4) FPN was significantly higher than CON on between-network deviation $\left(t_{(49)}=4.88, p=0.000012\right.$; see prior results sections for FPN vs CON comparisons on GVC and deviation scores related to Figure $11 \mathrm{~A}$, where all regions were included). This pattern of results replicated in the replication dataset: (1) FPN was 
A
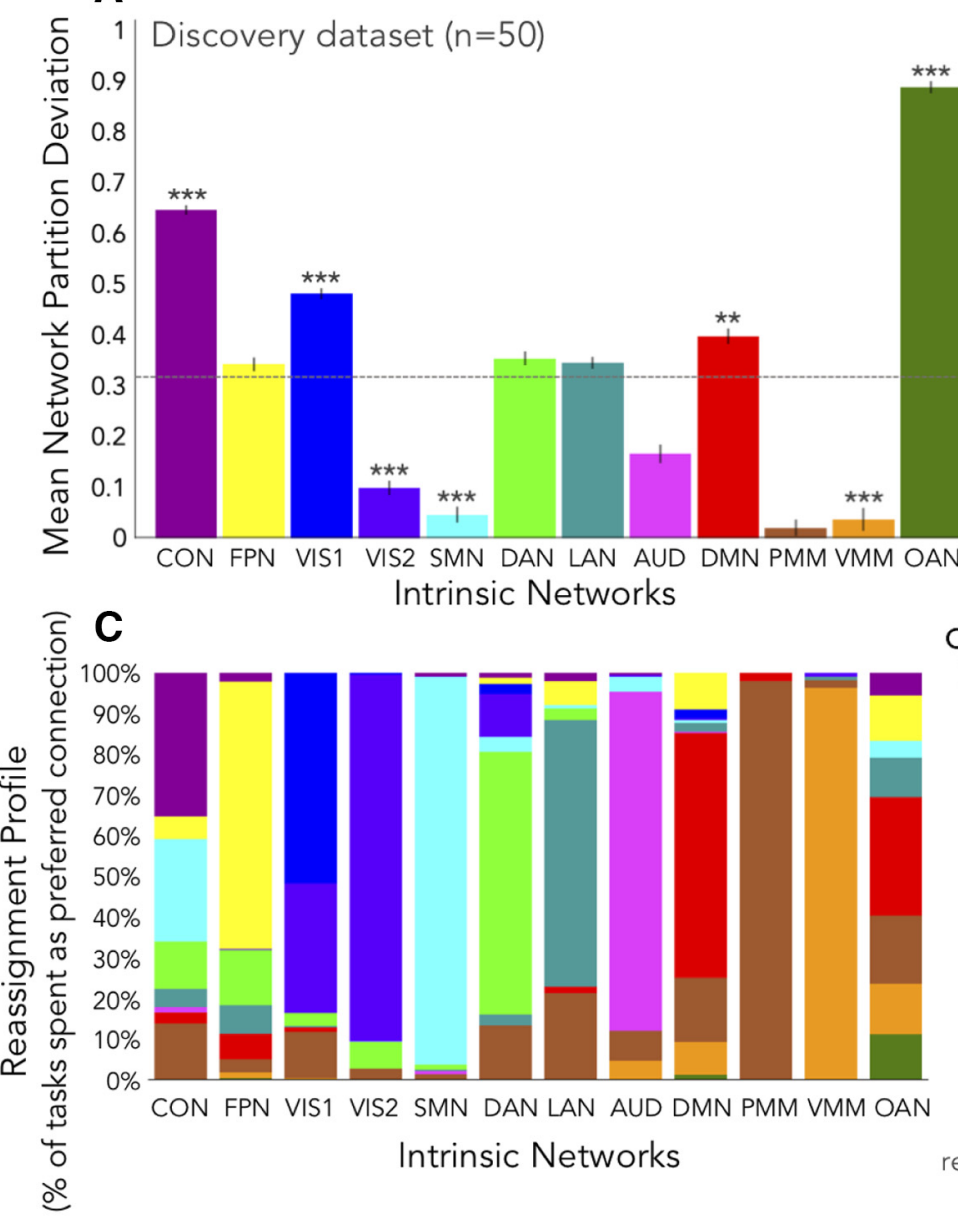

$\mathbf{E}$

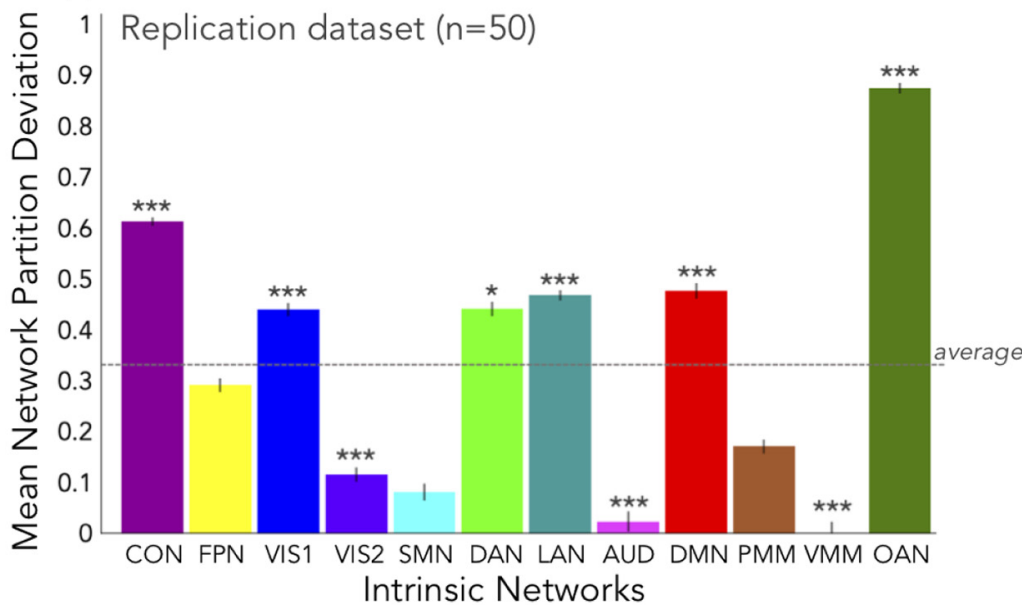

B

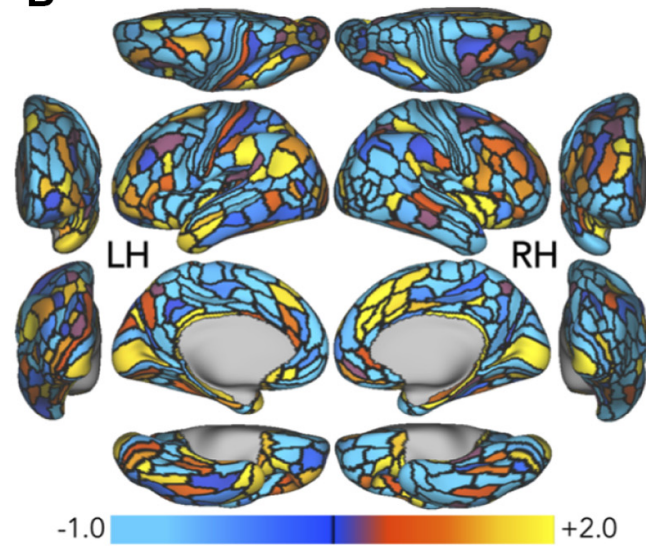

$\bar{x}$ Regional Deviation (normalized)

Connecting

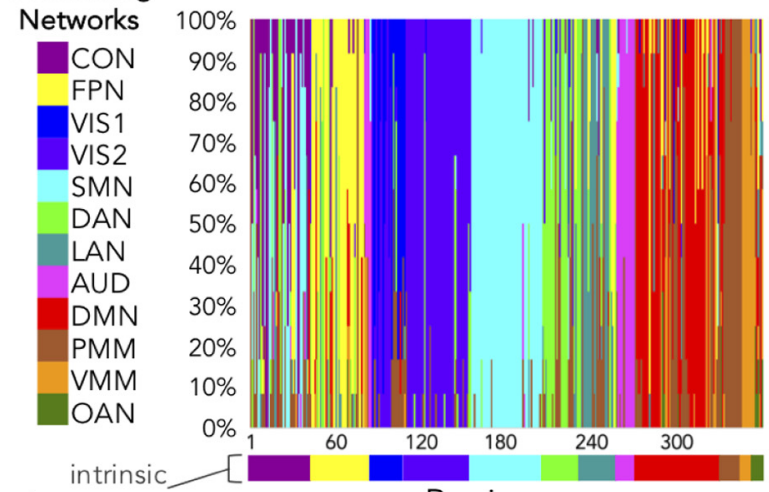

reference partition
Regions

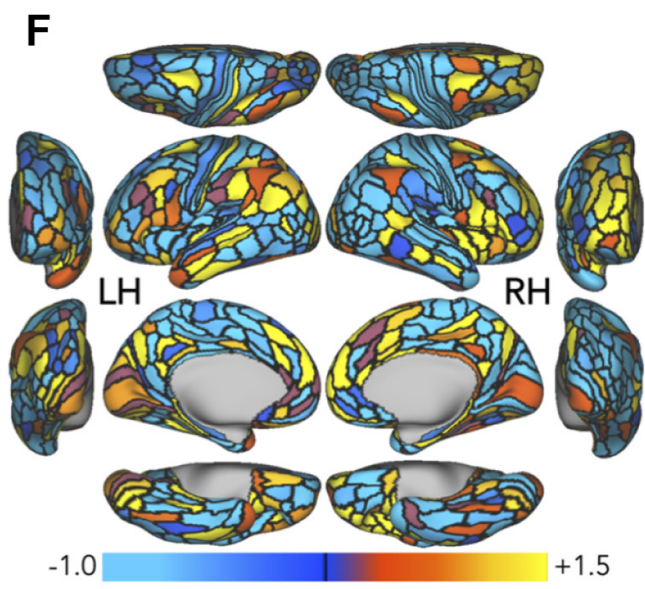

$\bar{x}$ Regional Deviation (normalized)

Figure 10. Network partition deviation. Mean, error bars, and hypothesis testing specifications were the same as in Figure 7. Asterisks: statistically significant $t$ tests, using the max-T approach (see Materials and Methods). ${ }^{*} p<0.05 ;{ }^{* *} p<0.005 ;{ }^{* * *} p<0.0005$. $A$, Network-mean deviation, discovery dataset. See Discussion for interpretations of the orbito-affective network (OAN). $\boldsymbol{B}$, Regional-mean deviation, discovery dataset. $\boldsymbol{C}$, Network-level reassignment profiles. For each intrinsic network ( $x$-axis), adherence (or 1-deviation) is depicted as the portion of the bar (connecting network) with the equivalent color. All other colors codify exactly which connecting networks were being preferred (see Table 3; Fig. 4) when deviating from the predefined partition. That is to say, deviation in $\boldsymbol{A}$ is expanded in $\boldsymbol{C}$ to show frequency of reassignments, across task states. $\boldsymbol{D}$, The same as in $\boldsymbol{C}$, but the $\boldsymbol{x}$-axis is depicted at the regional level (i.e., these regional reassignments were averaged to generate $\boldsymbol{C}$. $\boldsymbol{E}$, Network-mean deviation, replication dataset. $\boldsymbol{F}$, Regional-mean deviation, replication dataset. Deviation results highly overlapped between discovery $(\boldsymbol{A})$ and replication $(\boldsymbol{E})$ datasets: $R h 0=0.9580, p<0.00001$

significantly higher than CON on within-network GVC $\left(t_{(49)}=8.65, p<0.00001\right)$; (2) FPN was significantly higher than CON on between-network GVC $\left(t_{(49)}=10.26, p<0.00001\right)$; (3) CON was significantly higher than FPN on within-network deviation $\left(t_{(49)}=6.16, p<0.00001\right)$; and (4) FPN was significantly higher than CON on between-network deviation $\left(t_{(49)}=2.13\right.$, $p=0.03$ ).

To explore these results further, we created a color-coded graph of the partition reassignments captured by each version of deviation (Fig. 12). We used the Jaccard similarity index (see 

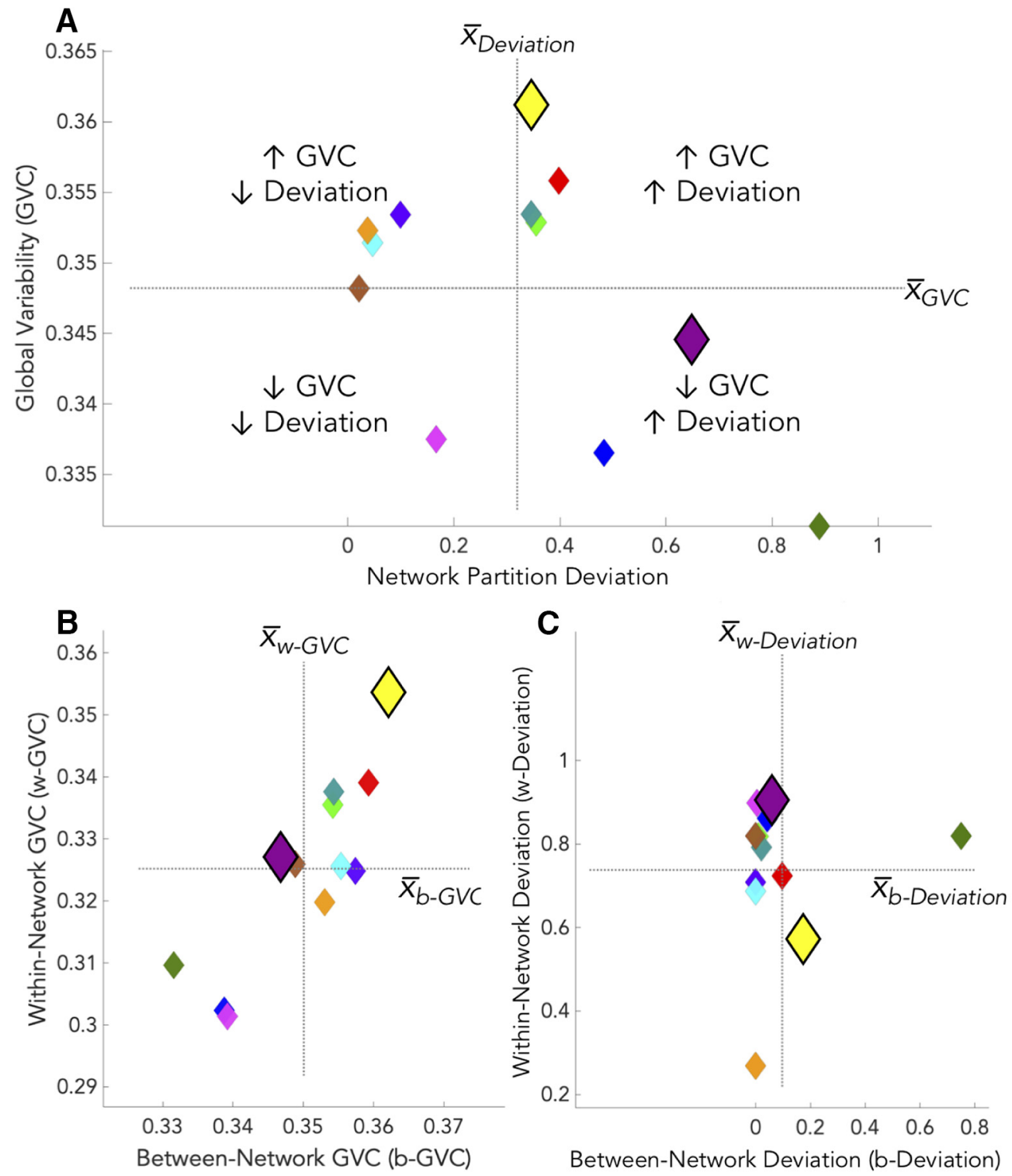

\section{CAB-NP Network Colors \\ Primary Visual \\ (VIS1) \\ Secondary Visual \\ (VIS2)}

Somatomotor

(SMN)

Cingulo-Opercular (CON)

\section{Dorsal Attention (DAN)}

\section{Language \\ (LAN)}

Frontoparietal (FPN)

\section{Auditory}

(AUD)

Default Mode

(DMN)

Posterior Multimodal (PMM)

Default Mode

(DMN)

Posterior Multimodal (PMM)

Figure 11. Cognitive control cartographies, discovery dataset. $\boldsymbol{A}$, GVC (as in Fig. 7A) plotted over deviation (as in Fig. 10A), with demarcation lines (dashed and crossed gray lines) indicating the cross-network mean for each dimension (all axes are centered at these marks for ease of viewing). This allowed us to "map out" multidimensional properties at once. For example, networks in the lower right quadrant of $\boldsymbol{A}$ (such as CON) exhibited GVC lower than the mean and deviation higher than the mean. This mapping suggests a nonlinear relationship between GVC and deviation, suggesting that each measure characterized a unique network property. In all panels, control network diamonds (FPN: yellow, CON: plum) are highlighted with dark black outlines and are larger than other network diamonds, with the sole visualization purpose of standing out as cognitive control networks. $\boldsymbol{B}$, GVC scores ( $y$-axis of panel $\boldsymbol{A}$ ) expanded by within-network and between-network values. $\boldsymbol{C}$, Deviation ( $x$-axis of panel $\boldsymbol{A}$ ) scores expanded by within-network and between-network values. The far-right legend depicts the (AB-NP color scheme (as in Fig. 3 ) used for the diamonds.

Materials and Methods) to quantify the relationship between reassignment patterns. We found FPN's between-network deviation (i.e., within-network connectivity held constant) was more similar to "all-data" deviation than its within-network deviation (i.e., between-network connectivity held constant): Jaccard indices of 0.29 and 0.18 , respectively (Fig. 12B). Yet, CON's withinnetwork deviation was more similar to all-data deviation than its between-network deviation (Jaccard indices of 0.31 and 0.16 , respectively; Fig. 12A). Supporting dissociation of FPN and CON network dynamics, the Jaccard similarity indices for CON and FPN were significantly different (Jaccard for deviation all-data and deviation within-network data, CON vs FPN: $t_{(49)}=3.30$, $p=0.0018$; Jaccard for deviation all-data and deviation betweennetwork data, CON vs FPN: $\left.t_{(49)}=-4.61, p=0.00003\right)$.
This result supports the conclusion that the high deviation exhibited by the CON was driven by its within-network connections, indicating that CON task-related dynamics were driven mostly by reduction in within-network intrinsic connectivity (network "disbanding") to increase the strength of between-network connections relative to (now-reduced) within-network connections. In contrast, FPN regions maintained their within-network connection patterns while varying their between-network connection patterns across rest and task. This is consistent with FPN maintaining its intrinsic within-network connectivity while reconfiguring its between-network connections in a task-specific manner. Moreover, the pattern of CON within-network decreases was task specific (Figs. $7 E, 8,12$ ). This is consistent with CON being a flexible hub network like FPN, but with a distinct mechanism involving 
A Deviation Input: All Data

Deviation Input: Within-Net Data Deviation Input: Between-Net Data

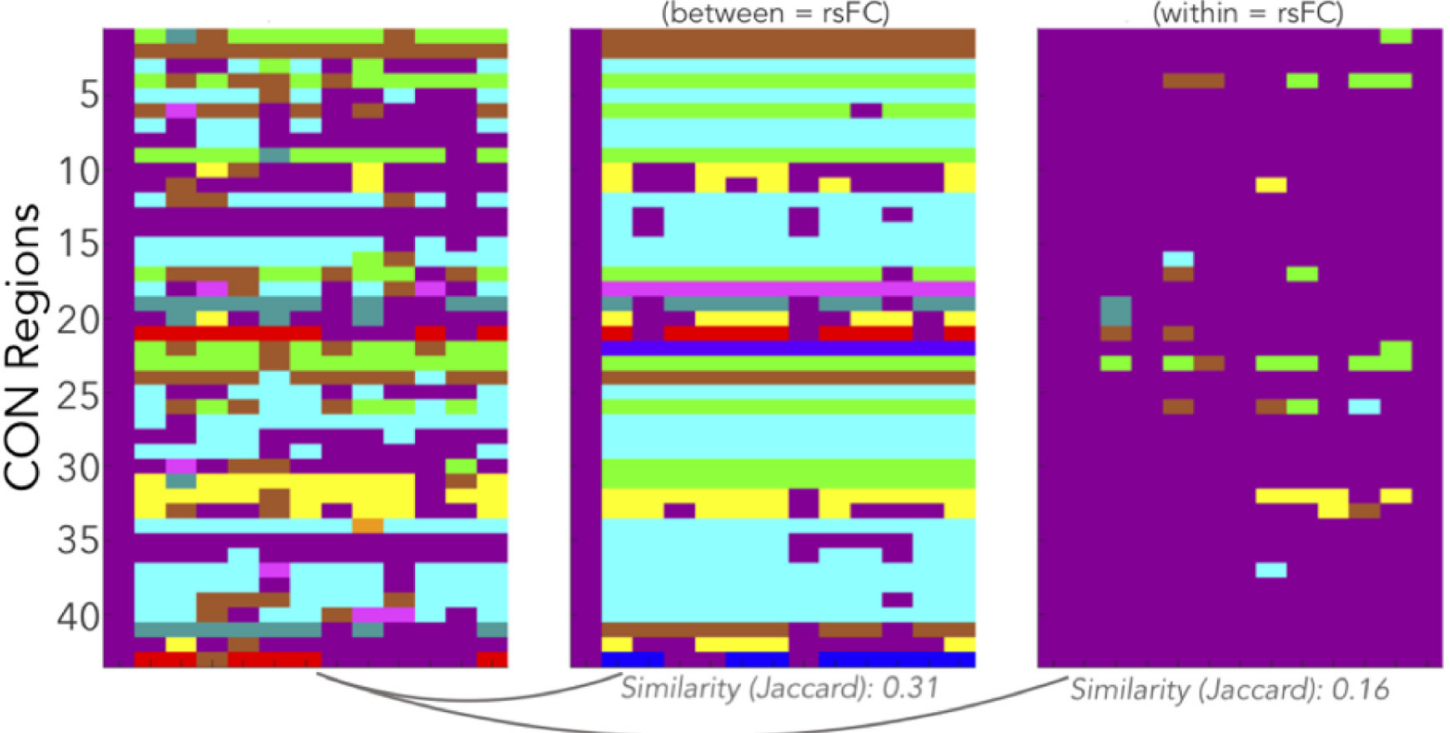

B
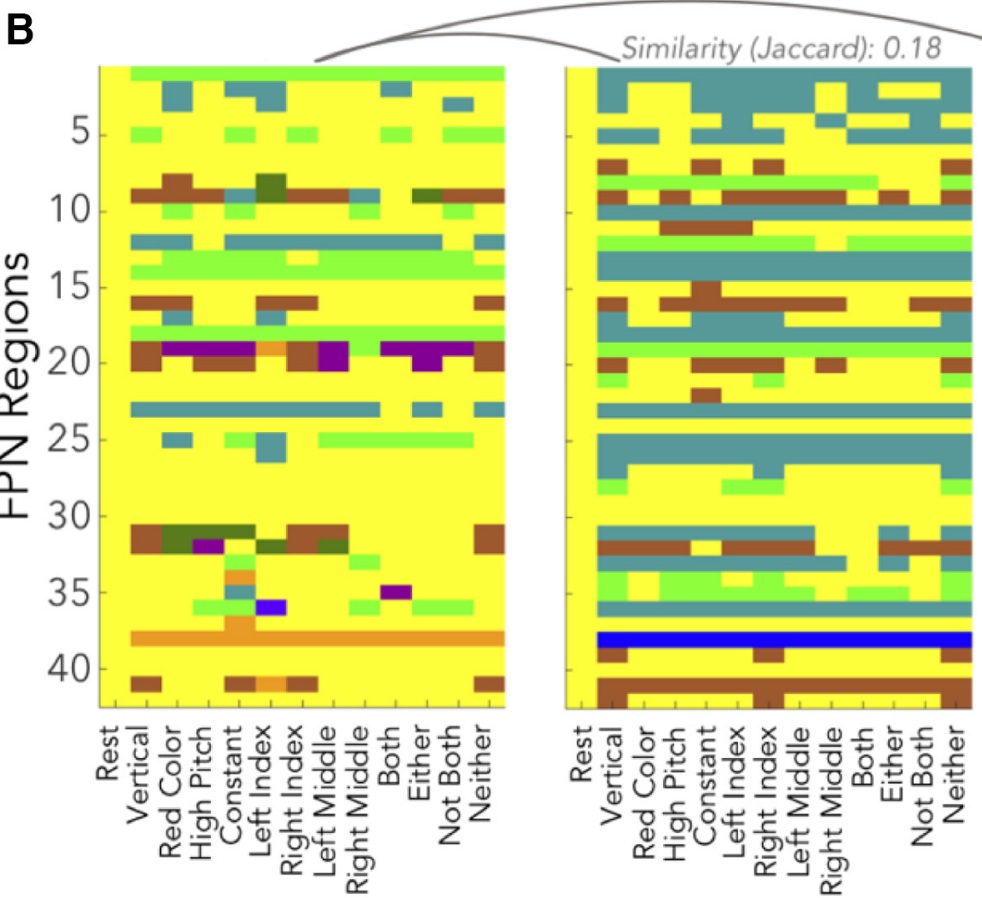

Similarity (Jaccard): 0.29
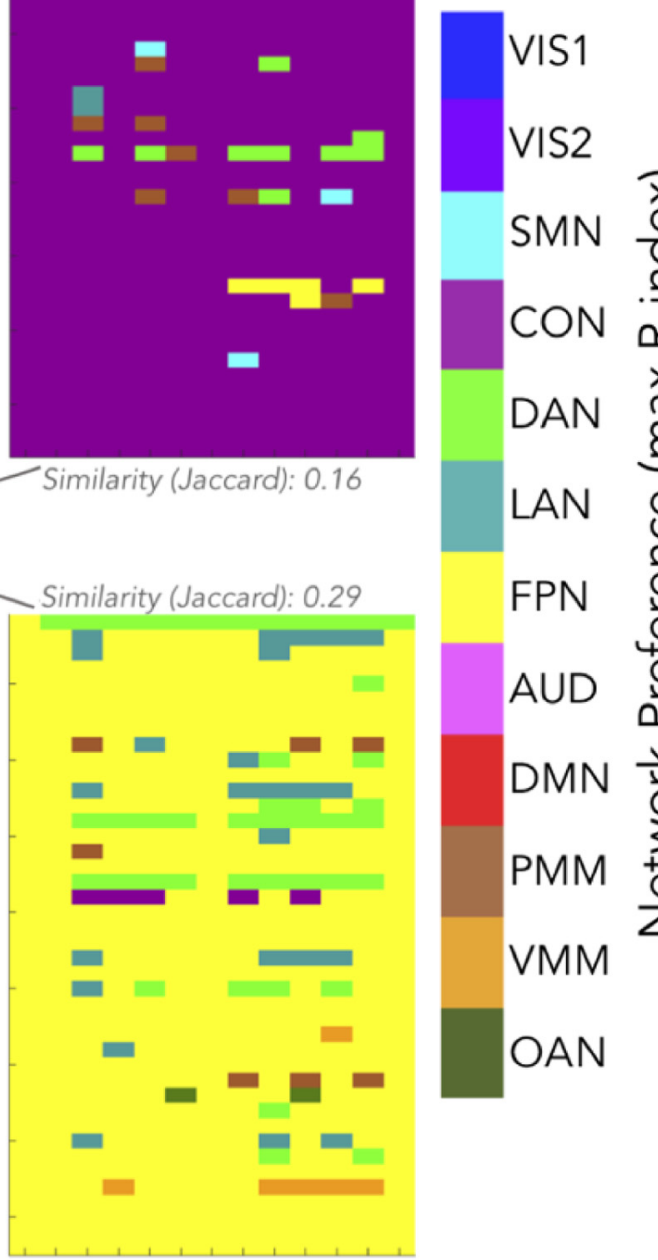

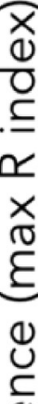

FPN
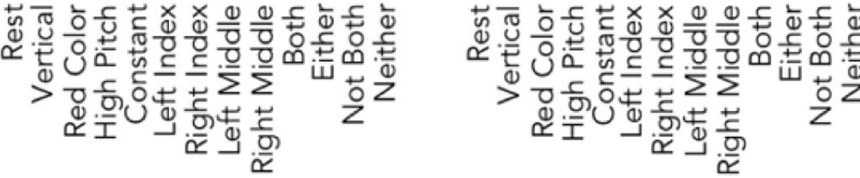

\section{C-PRO Rule Sets}

Figure 12. Reassignments conferred by the variants of network partition deviation in the control cartographies. Reassignment is the network index (based on the intrinsic partition) of the highest mean connectivity estimate, per task state. In each panel, Jaccard indices are listed to indicate similarity between two partitions. Network assignments are color coded (as in Fig. 3), and the 64 C-PRO task states are collapsed into 12 rule sets (plus Rest as a reference on each $x$-axis). $\boldsymbol{A}$, left, Network reassignments of CON regions from the deviation algorithm, with all connectivity data included in the input (Fig. 11A, $x$-axis). Middle, Within-network CON estimates used in the deviation algorithm (Fig. 11C, $y$-axis; see Fig. 5C). The Jaccard similarity of within-network and all-data deviation is 0.31 . Right, Between-network CON estimates used in the deviation algorithm (Fig. 11C, $x$-axis; see Fig. 5D). The Jaccard similarity of between-network and all-data deviation is 0.16 , which is lower than the within-network similarity to all-data. $\boldsymbol{B}$, Same as panel $\boldsymbol{A}$, except for FPN regions. The Jaccard similarity scores are: within-to-all $=0.18$, between-to-all $=0.29$. Thus, FPN showed the reverse pattern to CON, where between-network deviation is more similar to all-data deviation than within-network deviation.

"switching" from within-network to out-of-network connectivity via dynamic reduction of within-CON connectivity.

\section{Discussion}

The chief conclusion of the current study was that combining network science measures into network cartographies (multidimensional functional "mappings") allowed us to characterize cognitive control brain systems as either flexible coordinators (frontoparietal regions) or flexible switchers (cingulo-opercular regions). Network cartographies consisted of two primary dimensions: (1) GVC, which measures global FC reconfiguration across task states in a continuous manner; and (2) deviation, which measures global FC reconfiguration from rest to task in a discrete manner (Fig. 11). We found that FPN exhibited high GVC but low deviation, while CON showed the opposite pattern, consistent with complementary mechanisms of cognitive control. FPN appeared 
to act as a flexible coordinator, based on its extensive between-network FC reconfiguration along with maintenance of its within-network connections across rest and task states. In contrast, CON appeared to act as a flexible switcher, based on extensively reducing its within-network connections from rest to task to effectively switch to other networks during tasks (Fig. 2).

The present findings are broadly consistent with the view proposed by Dosenbach et al. (2006, 2007, 2008), which posited, based on fMRI task activations and rsFC, that control networks implement dissociable mechanisms. We used tFC along with dynamic graph-theoretic measures to expand on Dosenbach et al. In that work, FPN regions enacted control in a manner described as "active, adaptive, and online." The high tFCbased global variability we observed in FPN regions is consistent with adaptive monitoring and adjustment important for controlled processing (Cole and Schneider, 2007; Sadaghiani and D'Esposito, 2015; Crittenden et al., 2016). In contrast to FPN, Dosenbach et al. $(2006,2007,2008)$ proposed that CON underlies "stable set maintenance, task mode, and strategy" (also shown by Vaden et al., 2013). While we did find CON connectivity changes to be more consistent (across task states) than FPN (Fig. 7), we propose that its functional switching relates to the biased competition model put forth by Desimone et al. (1990) and Desimone and Duncan (1995) and related theories, as described below.

The biased competition model posits that neural representations compete for resources, such that stimuli, actions, and/or thoughts compete for attention during task performance (Desimone and Duncan, 1995). The theory suggests that competition is biased by top-down goal-related signals from prefrontal cortex and related areas (i.e., control networks). These top-down control signals are thought to shift the competition in bottom-up processing (e.g., in visual cortex), such that goal-relevant processes become more salient and more likely to "win." For instance, a top-down control signal could bias color-naming representations over word-reading representations to aid in Stroop task performance. This theory was built on by the guided activation theory (Miller and Cohen, 2001) and flexible hub theory (Cole et al., 2013b). In line with these later theories, we recently posited that such top-down biases to bottom-up competitive processes are especially important for RITL paradigms (such as the C-PRO task), and that they are implemented by tFC changes from control networks (Cole et al., 2017). In the present study, connectivity patterns of FPN and CON regions significantly decoded CPRO task rules (Fig. 8), suggesting that distributed interactions implemented by cognitive control networks critically support task representations. Further, network science measures probing those interactions suggested that top-down biases are implemented via two complementary mechanisms.

First, CON regions appeared to reduce their within-network connectivity and flexibly switch to other networks in a taskdependent manner. We observed this switching to occur with a relatively uniform distribution, across tasks and switched-to networks (Figs. 7, 10C), and with high deviation across tasks (Fig. $10 A$ ). We posit that CON transiently disbands and switches networks to lend resources ("weight" or "energy") to help goal-relevant regions/networks (e.g., visual and motor regions during visuo-motor tasks) win competitions with other regions/networks (or representations). Importantly, we propose that CON's switching property specifically helps win competitions by reducing functional interference from goal-irrelevant systems, such as interference with distracting stimuli or among goal-relevant representations. Second, FPN regions appeared to flexibly coordinate their global patterns of goal-driven biases with each other via maintaining within-network connectivity. This likely facilitates the coordination of complex task sets via facilitating interactions among combinations of task representations. This account illustrates a fundamental trade-off in controlled processing: implementing goal-relevant "programs" by FPN through coordinated (but potentially interfering) top-down biases, versus lending of resources via independent (and therefore less likely to interfere) top-down biases by CON to help goal-relevant brain systems win competitions.

Interestingly, FPN's between-network connectivity patterns were variable, but FPN's within-network intrinsic configuration remained intact across task states (Figs. 2, 12). On the one hand, between-network FC variability corroborates the notion that FPN supports task-specific coding (Crittenden et al., 2016) and selective attention demands (Sadaghiani and D'Esposito, 2015). On the other hand, within-network FC preservation suggests that FPN regions are coordinating the FC changes across FPN regions. These dynamics are well suited to address the "variable binding" problem (Feldman, 2013), where variable stimulus information must be linked to task rules to enact cognitive computations. In C-PRO tasks, variable rules must link via logical operations to perform a given task, and variable stimuli must link to those rules to produce correct behavior (Fig. 1). The maintenance of FPN's intrinsic organization combined with between-network reconfigurations, suggests a coding process that includes FPN, along with other, task-specific regions. This computational format would allow for variable stimulus information to be bound on a task-to-task basis. Specifically, we propose that FPN's role in this scheme is to flexibly coordinate task-specific coding. Notably, this would impose high processing demands on FPN regions, which we suggest to be facilitated by the CON freeing up resources, pointing to a computational trade-off across these two cognitive control networks.

Two other networks joined CON in having low variability and high deviation: the OAN and the primary visual network (VIS1). OAN had both the lowest GVC and the highest deviation of all networks. This suggests that the switching mechanism proposed for CON also occurs for OAN, although other studies suggest distinct functionality for OAN. Anatomically, OAN is localized to a small number of regions, ventromedial prefrontal cortex and nearby subcortical regions (Ji et al., 2019). Human lesion studies and animal models suggest a core role of OAN in emotion processing and value representation (Roy et al., 2012), and likely receives direct dopaminergic projections from the ventral tegmental area (Seamans and Yang, 2004). Emotion processing may seem counter to the non-emotional C-PRO paradigm, yet evidence from human lesion studies (Koenigs et al., 2007) and neuroimaging (Botvinick and Braver, 2015) demonstrates that emotion, in the form of motivation, biases competition between outcomes during complex decisions. Future research should assess whether OAN provides a similar mechanism to CON for top-down biasing, but via an emotional/motivational mode of processing. In contrast to OAN and CON, VIS1 was not diverse in its connectivity switches, primarily switching to the secondary visual network (extrastriate cortex; Fig. 10C,D), consistent with integrated processing across the two visual networks.

Aside from the high-variability/low-deviation and low-variability/high-deviation cartographic mappings of the FPN and CON, respectively (Fig. 11A), there were two other scenarios possible. First, high-variability and high-deviation across task states: networks exhibiting this profile would have fluctuating 
connectivity as well as notable partition reassignment from rest to task. The default network (Fig. 11A, red diamond) appeared to be the only network trending in this direction. Second, lowvariability and low-deviation across task states: networks exhibiting this profile would have stable connectivity estimates and adhere to their intrinsic partition. The auditory network was the only observed herein (Fig. 11A, pink diamond), which may relate to sensory regions' proposed "rigid core" organization (Bassett et al., 2013b). Other sensory-motor networks had low deviation compared with cognitive networks, suggesting low versus high deviation was indicative of sensory-motor versus cognitive network properties. Future work is warranted to explore this, particularly if diverse task paradigms are implemented.

An essential consideration for future studies regards the question of timescale. Dosenbach et al. $(2007,2008)$ found increases in CON activity to be sustained across tasks, while FPN activations were present at task onset then adaptively varied with changing task demands. In the present work, we applied network metrics across a set of dynamic task states demanding high levels of cognitive control. While measures of global variability summarized varying connectivity patterns across states, further examination could determine the timescales of control network mechanisms. Relatedly, future studies would benefit from considering how electrophysiological signatures of neural processing and network properties relate in terms of the instantiation of cognitive control. In resting-state based studies, Sadaghiani et al. (2010, 2012) found distinctions between FPN and CON based on alpha band signatures. Spontaneous CON activity related to increases in global power, while FPN related to increases in long-range phase synchrony. These signatures correspond to the functions of tonic alertness and phasic control, respectively. In future work, both rest and control-related task states should be assessed via alpha band signatures, as well as potential changes in those signatures from rest to task. A potential outcome is that increases in global power (CON) and long-range synchrony (FPN) would be more apparent from rest to task, constituting another "mappable" reconfiguration property of cognitive control. This would further support the proposition that $\mathrm{CON}$ is suited to lend processing resources, and FPN to adaptively integrate task-specific information. Moreover, possible interactions between the properties discovered herein (Fig. 11) and electrophysiological properties remains an empirical question.

Taken together, constructing a functional cartography by combining multiple network science measures allowed us to characterize FPN and CON as complementary systems of cognitive control. We demonstrated that FPN regions enacted control via flexible coordination of reconfiguring connectivity patterns, and $\mathrm{CON}$ regions enacted control via flexible switching of network affiliations to lend resources to task-relevant networks. All results replicated in a dataset with distinct subjects, and expanded prior theories that distinct mechanisms of cognitive control are instantiated in parallel via separate large-scale brain systems. Looking forward, we expect the dynamic network neuroscience approach expanded on here will be effective for functionally characterizing the relationship between neural and cognitive dynamics in other brain systems and other cognitive paradigms.

\section{References}

Amelio A, Tagarelli A (2017) Revisiting resolution and Inter-layer coupling factors in modularity for multilayer networks. Proceedings of the 2017 IEEE/ACM International Conference on Advances in Social Networks
Analysis and Mining 2017, pp 266-273 ASONAM '17. New York: Association for Computing Machinery.

Anderson ML, Magruder J (2017) Split-sample strategies for avoiding false discoveries. National Bureau of Economic Research Working Paper 23544

Bassett DS, Wymbs NF, Porter MA, Mucha PJ, Carlson JM, Grafton ST (2011) Dynamic reconfiguration of human brain networks during learning. Proc Natl Acad Sci USA 108:7641-7646.

Bassett DS, Porter MA, Wymbs NF, Grafton ST, Carlson JM, Mucha PJ (2013a) Robust detection of dynamic community structure in networks. Chaos 23:013142.

Bassett DS, Wymbs NF, Rombach MP, Porter MA, Mucha PJ, Grafton ST (2013b) Task-based core-periphery organization of human brain dynamics. PLoS Comput Biol 9:e1003171.

Blair RC, Karniski W (1993) An alternative method for significance testing of waveform difference potentials. Psychophysiology 30:518-524.

Botvinick MM (2007) Conflict monitoring and decision making: reconciling two perspectives on anterior cingulate function. Cogn Affect Behav Neurosci 7:356-366.

Botvinick M, Braver T (2015) Motivation and cognitive control: from behavior to neural mechanism. Annu Rev Psychol 66:83-113.

Braem S, Bugg JM, Schmidt JR, Crump MJC, Weissman DH, Notebaert W, Egner T (2019) Measuring adaptive control in conflict tasks. Trends Cogn Sci 23:769-783.

Braun U, Schäfer A, Walter H, Erk S, Romanczuk-Seiferth N, Haddad L, Schweiger JI, Grimm O, Heinz A, Tost H, Meyer-Lindenberg A, Bassett DS (2015) Dynamic reconfiguration of frontal brain networks during executive cognition in humans. Proc Natl Acad Sci USA 112:11678-11683.

Chen M, Kuzmin K, Szymanski BK (2015) Community detection via maximization of modularity and its variants. arXiv 1507.00787.

Cohen MR, Maunsell JHR (2009) Attention improves performance primarily by reducing interneuronal correlations. Nat Neurosci 12:1594-1600.

Cole MW, Schneider W (2007) The cognitive control network: integrated cortical regions with dissociable functions. Neuroimage 37:343-360.

Cole MW, Yeung N, Freiwald WA, Botvinick M (2009) Cingulate cortex: diverging data from humans and monkeys. Trends Neurosci 32:566-574.

Cole MW, Bagic A, Kass R, Schneider W (2010) Prefrontal dynamics underlying rapid instructed task learning reverse with practice. J Neurosci 30:14245-14254.

Cole MW, Laurent P, Stocco A (2013a) Rapid instructed task learning: a new window into the human brain's unique capacity for flexible cognitive control. Cogn Affect Behav Neurosci 13:1-22.

Cole MW, Reynolds JR, Power JD, Repovs G, Anticevic A, Braver TS (2013b) Multi-task connectivity reveals flexible hubs for adaptive task control. Nat Neurosci 16:1348-1355.

Cole MW, Bassett DS, Power JD, Braver TS, Petersen SE (2014) Intrinsic and task-evoked network architectures of the human brain. Neuron 83:238251.

Cole MW, Braver TS, Meiran N (2017) The task novelty paradox: flexible control of inflexible neural pathways during rapid instructed task learning. Neurosci Biobehav Rev 81:4-15.

Cole MW, Ito T, Schultz D, Mill R, Chen R, Cocuzza C (2019) Task activations produce spurious but systematic inflation of task functional connectivity estimates. Neuroimage 189:1-18.

Crittenden BM, Mitchell DJ, Duncan J (2016) Task encoding across the multiple demand cortex is consistent with a frontoparietal and cingulo-opercular dual networks distinction. J Neurosci 36:6147-6155.

Desimone R, Duncan J (1995) Neural mechanisms of selective visual attention. Annu Rev Neurosci 18:193-222.

Desimone R, Wessinger M, Thomas L, Schneider W (1990) Attentional control of visual perception: cortical and subcortical mechanisms. Cold Spring Harb Symp Quant Biol 55:963-971.

Dosenbach NUF, Visscher KM, Palmer ED, Miezin FM, Wenger KK, Kang HC, Burgund ED, Grimes AL, Schlaggar BL, Petersen SE (2006) A core system for the implementation of task sets. Neuron 50:799-812.

Dosenbach NUF, Fair DA, Miezin FM, Cohen AL, Wenger KK, Dosenbach RAT, Fox MD, Snyder AZ, Vincent JL, Raichle ME, Schlaggar BL, Petersen SE (2007) Distinct brain networks for adaptive and stable task control in humans. Proc Natl Acad Sci USA 104:11073-11078.

Dosenbach NUF, Fair DA, Cohen AL, Schlaggar BL, Petersen SE (2008) A dual-networks architecture of top-down control. Trends Cogn Sci 12:99105 . 
Feldman J (2013) The neural binding problem(s). Cogn Neurodyn 7:1-11.

Fuster JM, Bauer RH, Jervey JP (1985) Functional interactions between inferotemporal and prefrontal cortex in a cognitive task. Brain Res 330:299307.

Gershon RC, Wagster MV, Hendrie HC, Fox NA, Cook KF, Nowinski CJ (2013) NIH toolbox for assessment of neurological and behavioral function. Neurology 80:S2-S6.

Glasser MF, Sotiropoulos SN, Wilson JA, Coalson TS, Fischl B, Andersson JL, Xu J, Jbabdi S, Webster M, Polimeni JR, Van Essen DC, Jenkinson M; WU-Minn HCP Consortium (2013) The minimal preprocessing pipelines for the Human Connectome Project. Neuroimage 80:105-124.

Glasser MF, Coalson TS, Robinson EC, Hacker CD, Harwell J, Yacoub E, Ugurbil K, Andersson J, Beckmann CF, Jenkinson M, Smith SM, Van Essen DC (2016) A multi-modal parcellation of human cerebral cortex. Nature 536:171-178.

Glerean E, Pan RK, Salmi J, Kujala R, Lahnakoski JM, Roine U, Nummenmaa L, Leppämäki $S$, Nieminen-von Wendt T, Tani $P$, Saramäki J, Sams M, Jääskeläinen IP (2016) Reorganization of functionally connected brain subnetworks in high-functioning autism. Hum Brain Mapp 37:1066-1079.

Gordon EM, Laumann TO, Adeyemo B, Huckins JF, Kelley WM, Petersen SE (2016) Generation and evaluation of a cortical area parcellation from resting-state correlations. Cereb Cortex 26:288-303.

Guimerà R, Nunes Amaral LA (2005) Cartography of complex networks: modules and universal roles. J Stat Mech 2005:nihpa35573.

Haxby JV, Gobbini MI, Furey ML, Ishai A, Schouten JL, Pietrini P (2001) Distributed and overlapping representations of faces and objects in ventral temporal cortex. Science 293:2425-2430.

He BJ (2013) Spontaneous and task-evoked brain activity negatively interact. J Neurosci 33:4672-4682.

Ito T, Kulkarni KR, Schultz DH, Mill RD, Chen RH, Solomyak LI, Cole MW (2017) Cognitive task information is transferred between brain regions via resting-state network topology. Nat Commun 8:1027.

Ito T, Brincat SL, Siegel M, Mill RD, He BJ, Miller EK, Rotstein HG, Cole MW (2019) Task-evoked activity quenches neural correlations and variability in large-scale brain systems. bioRxiv 560730. doi: https://doi.org/ $10.1101 / 560730$

Ji JL, Spronk M, Kulkarni K, Repovš G, Anticevic A, Cole MW (2019) Mapping the human brain's cortical-subcortical functional network organization. Neuroimage 185:35-57.

Koenigs M, Young L, Adolphs R, Tranel D, Cushman F, Hauser M, Damasio A (2007) Damage to the prefrontal cortex increases utilitarian moral judgements. Nature 446:908-911.

Krienen FM, Yeo BTT, Buckner RL (2014) Reconfigurable task-dependent functional coupling modes cluster around a core functional architecture. Phil Trans R Soc Lond B Biol Sci 369:20130526.

Mantel N (1967) The detection of disease clustering and a generalized regression approach. Cancer Res 27:209-220.

Marcus DS, Harwell J, Olsen T, Hodge M, Glasser MF, Prior F, Jenkinson M, Laumann T, Curtiss SW, Van Essen DC (2011) Informatics and data mining tools and strategies for the human connectome project. Front Neuroinform 5:4.

Mattar MG, Cole MW, Thompson-Schill SL, Bassett DS (2015) A functional cartography of cognitive systems. PLoS Comput Biol 11:e1004533.

Medaglia JD, Lynall ME, Bassett DS (2015) Cognitive network neuroscience. J Cogn Neurosci 27:1471-1491.

Miller EK, Cohen JD (2001) An integrative theory of prefrontal cortex function. Annu Rev Neurosci 24:167-202.

Mucha PJ, Richardson T, Macon K, Porter MA, Onnela JP (2010) Community structure in time-dependent, multiscale, and multiplex networks. Science 328:876-878.

Mur M, Bandettini PA, Kriegeskorte N (2009) Revealing representational content with pattern-information fMRI-an introductory guide. Soc Cogn Affect Neurosci 4:101-109.
Murphy K, Birn RM, Handwerker DA, Jones TB, Bandettini PA (2009) The impact of global signal regression on resting state correlations: are anticorrelated networks introduced?. Neuroimage 44:893-905.

Nichols TE, Holmes AP (2002) Nonparametric permutation tests for functional neuroimaging: a primer with examples. Hum Brain Mapp 15:1-25.

Norman-Haignere SV, McCarthy G, Chun MM, Turk-Browne NB (2012) Category-selective background connectivity in ventral visual cortex. Cereb Cortex 22:391-402.

Ponce-Alvarez A, He BJ, Hagmann P, Deco G (2015) Task-driven activity reduces the cortical activity space of the brain: experiment and wholebrain modeling. PLoS Comput Biol 11:e1004445.

Power JD, Cohen AL, Nelson SM, Wig GS, Barnes KA, Church JA, Vogel AC, Laumann TO, Miezin FM, Schlaggar BL, Petersen SE (2011) Functional network organization of the human brain. Neuron 72:665678.

Power JD, Schlaggar BL, Lessov-Schlaggar CN, Petersen SE (2013) Evidence for hubs in human functional brain networks. Neuron 79:798-813.

Roy M, Shohamy D, Wager TD (2012) Ventromedial prefrontal-subcortical systems and the generation of affective meaning. Trends Cogn Sci 16:147-156.

Sadaghiani S, D'Esposito M (2015) Functional characterization of the cingulo-opercular network in the maintenance of tonic alertness. Cereb Cortex 25:2763-2773.

Sadaghiani S, Scheeringa R, Lehongre K, Morillon B, Giraud A-L, Kleinschmidt A (2010) Intrinsic connectivity networks, alpha oscillations, and tonic alertness: a simultaneous electroencephalography/functional magnetic resonance imaging study. J Neurosci 30:10243-10250.

Sadaghiani S, Scheeringa R, Lehongre K, Morillon B, Giraud A-L, D’Esposito M, Kleinschmidt A (2012) $\alpha$-band phase synchrony is related to activity in the fronto-parietal adaptive control network. J Neurosci 32:1430514310 .

Schneider W, Chein JM (2003) Controlled and automatic processing: behavior, theory, and biological mechanisms. Cogn Sci 27:525-559.

Schneider W, Eschman A, Zuccolotto A (2002) E-Prime user's guide. Pittsburgh: Psychology Software Tools.

Schultz DH, Ito T, Solomyak LI, Chen RH, Mill RD, Anticevic A, Cole MW (2019) Global connectivity of the fronto-parietal cognitive control network is related to depression symptoms in the general population. Netw Neurosci 3:107-123.

Seamans JK, Yang CR (2004) The principal features and mechanisms of dopamine modulation in the prefrontal cortex. Prog Neurobiol 74:1-58.

Seeley WW, Menon V, Schatzberg AF, Keller J, Glover GH, Kenna H, Reiss AL, Greicius MD (2007) Dissociable intrinsic connectivity networks for salience processing and executive control. J Neurosci 27:2349-2356.

Sporns O, Betzel RF (2016) Modular brain networks. Annu Rev Psychol 67:613-640.

Vaden KI Jr, Kuchinsky SE, Cute SL, Ahlstrom JB, Dubno JR, Eckert MA (2013) The cingulo-opercular network provides word-recognition benefit. J Neurosci 33:18979-18986.

Varoquaux G, Raamana PR, Engemann DA, Hoyos-Idrobo A, Schwartz Y, Thirion B (2017) Assessing and tuning brain decoders: cross-validation, caveats, and guidelines. Neuroimage 145:166-179.

Waskom ML, Kumaran D, Gordon AM, Rissman J, Wagner AD (2014) Frontoparietal representations of task context support the flexible control of goal-directed cognition. J Neurosci 34:10743-10755.

Yeo BTT, Krienen FM, Sepulcre J, Sabuncu MR, Lashkari D, Hollinshead M, Roffman JL, Smoller JW, Zöllei L, Polimeni JR, Fischl B, Liu H, Buckner RL (2011) The organization of the human cerebral cortex estimated by intrinsic functional connectivity. J Neurophysiol 106:1125-1165.

Yeo BTT, Krienen FM, Eickhoff SB, Yaakub SN, Fox PT, Buckner RL, Asplund CL, Chee MWL (2015) Functional specialization and flexibility in human association cortex. Cereb Cortex 25:3654-3672. 